\title{
Rosaceae, Brassicaceae and pollen beetles: exploring relationships and evolution in an anthophilous beetle lineage (Nitidulidae, Meligethes-complex of genera) using an integrative approach
}

Meike Liu ${ }^{1,2}$, Min Huang ${ }^{1 *}$, Andrew Richard Cline ${ }^{3}$, Emiliano Mancini ${ }^{4}$, Andrea Scaramuzzi ${ }^{4}$, Simone Paradisi ${ }^{4}$, Paolo Audisio ${ }^{4}$, Davide Badano ${ }^{4}$ and Simone Sabatelli $i^{4^{*}}$ (D)

\begin{abstract}
Background: Meligethes are pollen-beetles associated with flowers of Rosaceae as larvae. This genus currently consists of 63 known species in two subgenera, Meligethes and Odonthogethes, predominantly occurring in the eastern Palaearctic. We analyzed 74 morphological and ecological characters (169 states) of all species, as well as of 11 outgroup species from 7 Meligethinae genera (including Brassicogethes), to investigate their phylogeny. We also conducted a parallel molecular analysis on 9 Meligethes, 9 Odonthogethes, 3 Brassicogethes and 2 Meligethinus species based on DNA sequence data from mitochondrial (COI, 16S) and nuclear (CAD) genes.

Results: Morphological phylogenetic reconstructions supported the monophyly of the whole genus and clades corresponding to purported subgenera Meligethes s.str. and Odonthogethes. Main species-groups were mostly confirmed, however some unresolved polytomies remained. Molecular data placed members of Brassicogethes (including 42 mostly W Palearctic species associated with Brassicaceae) as sister to Odonthogethes, with this clade being sister to Meligethes s.str. This phylogenetic scenario suggests that monophyletic Meligethes s.str., Odonthogethes and Brassicogethes should be regarded alternatively as three subgenera of a monophyletic Meligethes, or three genera in a monophyletic genus-complex, with mutually monophyletic Brassicogethes and Odonthogethes. Molecular analyses estimated the origin of this lineage at ca. 14-15 Mya from a common stem including Meligethinus.

(Continued on next page)
\end{abstract}

\footnotetext{
* Correspondence: huangmin@nwafu.edu.cn; simone.sabatelli@uniroma1.it

${ }^{1}$ Key Laboratory of Plant Protection Resources and Pest Management of Ministry of Education, Entomological Museum, Northwest A\&F University, Yangling, Xianyang, Shaanxi, China

${ }^{4}$ Dipartimento di Biologia e Biotecnologie "Charles Darwin", Sapienza Università di Roma, Rome, Italy

Full list of author information is available at the end of the article
} 


\begin{abstract}
(Continued from previous page)
Conclusions: We hypothesize that the ancestor of Meligethes specialized on Rosaceae in the Middle Miocene (likely in Langhian Age) and subsequently radiated during Late Miocene and Plio-Pleistocene maintaining a trophic niche on this plant family. This radiation was primarily due to geographic isolation in E Asiatic mountain systems. Combined evidence from morphology, ancestral state parsimony reconstruction of host-plant associations and molecular evidence suggested that Rosoideae (Rosa spp.) represented the ancestral hosts of Meligethes s.str., followed by an independent shift of ancestral Odonthogethes (ca. 9-15 Mya) on Rubus (Rosoideae) and members of Rosaceae Spiraeoideae. Other ancestral Odonthogethes probably shifted again on the unrelated plant family Brassicaceae (maybe 8-14 Mya in S China), allowing a rapid westward radiation of the Brassicogethes clade.
\end{abstract}

Keywords: Pollen beetles, Rosaceae, Brassicaceae, Evolution, Host-shift, Palaearctic region

\section{Background}

Nitidulidae, with almost 4500 known species, is a mid-sized family of the order Coleoptera. Within this taxon, the diverse subfamily Meligethinae comprises about 700 polleneating species described worldwide [1-4]. Recently, the classification of Meligethinae underwent numerous changes in light of both molecular and morphological evidence, restricting the concept to several genera, particularly in the case of the previously polyphyletic genus Meligethes Stephens, 1830 [2, 5, 6]. Meligethes, even as presently bounded [2] [Fig. 1a (a), (b)], is still a rather species-rich genus, consisting of more than 60 species predominantly occurring in the Eastern Palaearctic [7-10] (Fig. 1 b-c; Table 1). This group includes species that are all associated with flowers of Rosaceae as larvae [2]. Most of Meligethes are oligophagous, although some members appear strictly monophagous $[1,9,10]$. Some locally common Meligethes [e.g. $M$. atratus (Oliver, 1790), M. flavimanus Stephens, 1830, M. violaceus Reitter, 1873)] also represent economically significant potential pests, attacking blossoms of ornamental roses (Rosa spp.) and plum trees (Prunus spp.) in Europe, Japan, and elsewhere [1,11-13].

The closely related and purported sister genus Brassicogethes Audisio \& Cline, 2009 [Fig. 1 a (c), d], comprises instead some forty, mostly Western Palaearctic species, all associated with Brassicaceae [2, 5, 14-23], and a few species [e.g. B. aeneus (F.) and B. viridescens (F.)] represent economically important pests, massively attacking blossoms of oilseed rapes, broccoli, cauliflowers, and others.

Most species of Meligethes (and several within the related genus Brassicogethes) have been recently analyzed through an integrated approach combining morphological, molecular, and bionomical data on larval ecology $[2,5,14-25]$. These contributions were based on morphological and molecular data from adults and on larval bionomical data. The thus far available molecular data set for Meligethes and Brassicogethes, including sequences of three mitochondrial and nuclear genes for 23 species (Table 2), allowed depicting a first phylogenetic scenario of Meligethes and some other related genera, and also provided a framework for understanding the origin of this group of Meligethinae, its evolution on different subfamilies of Rosaceae, and, possibly, the shift of ancestral Brassicogethes to Brassicaceae.

Although the genus Meligethes was included in previous phylogenetic studies, those were aimed to resolve suprageneric classification using molecular approaches [24-26] and only a few widespread W-Palaearctic species were represented. Herein, we analyzed 69 morphological characters (with 157 character states) of adults (Fig. 5) for all 63 known Meligethes (s.l.) species (including a thus far undescribed species from $\mathrm{S}$ China), as well as 11 outgroup species belonging to 7 different related Meligethinae genera, including 3 Brassicogethes species. Five additional bionomical characters (with 12 character states) were also analyzed combining available (published and unpublished) data on Meligethes larval ecology. The present work, then, constitutes the first comprehensive phylogenetic analysis at the species level of this large group, with a focus on E Palaearctic lineages.

\section{The genus Meligethes Stephens, 1830}

As recently summarized [7], the genus Meligethes [Fig. 1 a (a), (b)] was formally established by Stephens in 1830 [27], based on the type species Nitidula rufipes Marsham, 1802 [present day valid name: Meligethes atratus (Olivier, 1790)]. A new preliminary phylogenetic scenario for Meligethes s.l., previously including a heterogeneous and polyphyletic mixture of taxa, formally comprising more than 500 species worldwide, was recently presented [2, 24-26]. In the former paper [2], which was based on morphology of adults and preliminary molecular data, 22 genera were described as new, and 6 previously recognized subgenera of Meligethes were elevated to generic rank, delimiting Meligethes to some thirty Palaearctic species that utilized Rosaceae as larval host-plants. Following the original descriptions of the few European species [27-30], several new or presumed new species were separately added from the Eastern Palaearctic and northern Oriental Regions by a long 


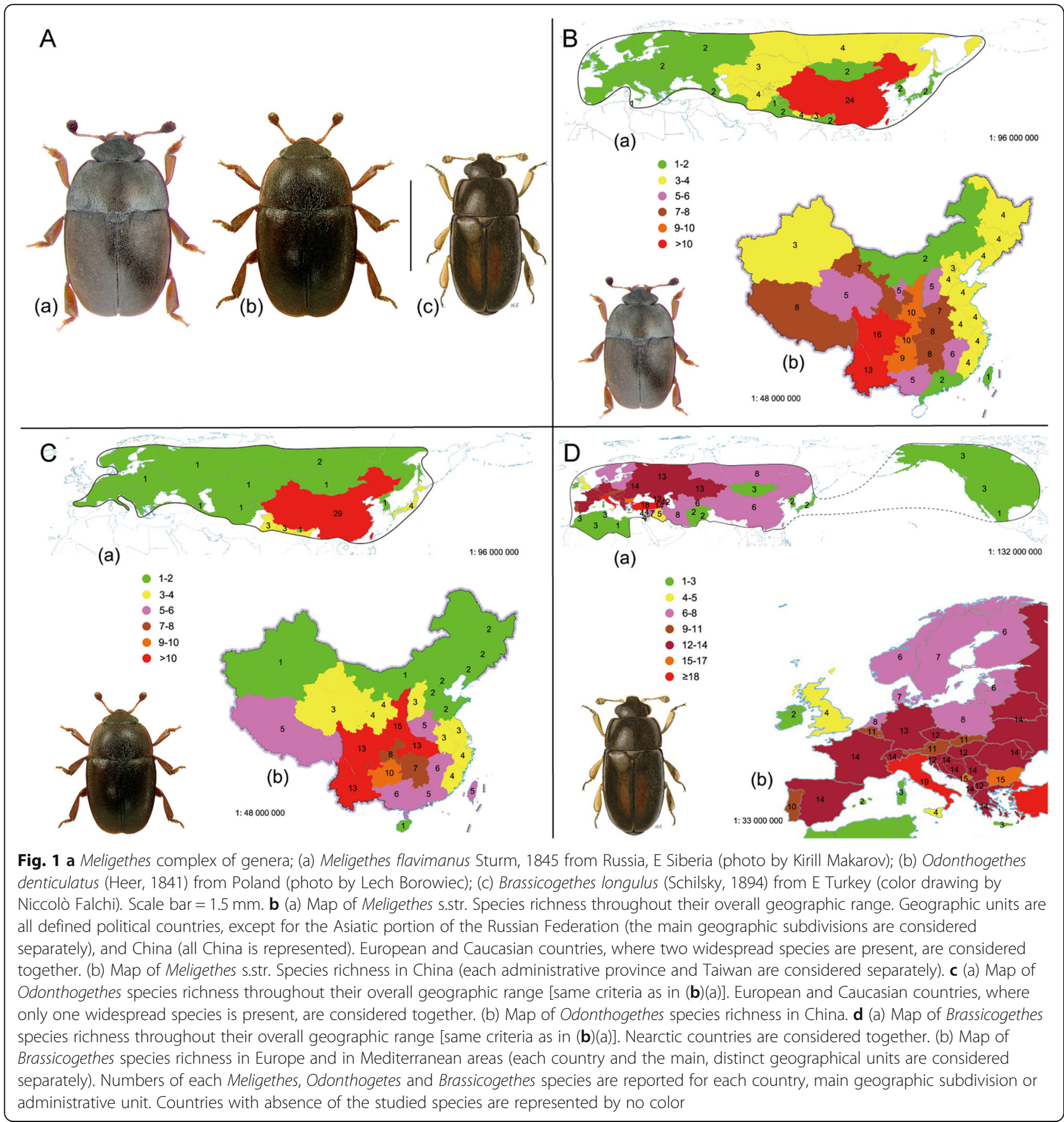

series of authors in the time span from 1845 to 1997 , including two important (although very preliminary) revisions [31, 32] of both purported subgenera. Two Chinese species were added [33, 34], before the revision of the whole genus Meligethes [7], as currently delimited [2], and 23 new species (21 from China) have been described. Following this revision [7], a few other new species from China were also added [8-10], and other potential new species are presently under scrutiny based on recently collected Chinese material. The genus, comprising a new species still waiting for a formal description, but considered in the present analysis $(M$. sp. cfr. Pectoralis from S China; Table 1, and Tables 3-4 in Additional files), now includes 63 species in two subgenera (Meligethes s.str. and Odonthogethes Reitter, 1871) [7-10] (Table 1).

Most Meligethes (s.l.) diversity occurs in the southern countries of the Eastern Palearctic and in the northern portions of the Oriental Region (Middle Asia, China, Japan, $\mathrm{N}$ Indian subcontinent); but China certainly 
Table 1 Summary of information on all 62 described species of Meligethes s.str. and Odonthogethes

\begin{tabular}{|c|c|c|c|c|c|c|c|}
\hline specific epithet & $\begin{array}{l}\text { author(s) and } \\
\text { year of } \\
\text { description }\end{array}$ & subgenus & $\begin{array}{l}\text { species } \\
\text { group }\end{array}$ & $\begin{array}{l}\text { host- } \\
\text { plant }\end{array}$ & distribution & type(s) & notes \\
\hline argentithorax & $\begin{array}{l}\text { Audisio, Sabatelli } \\
\text { \& Jelínek, } 2015\end{array}$ & Meligethes & $\begin{array}{l}\text { auripilis/ } \\
\text { binotatus }\end{array}$ & Rosa spp. & $\begin{array}{l}\text { W and SW China "http://encyclopedia2. } \\
\text { thefreedictionary.com/Qinghai"(Sichuan) }\end{array}$ & $\begin{array}{l}\text { CAR- } \\
\text { MZUR }\end{array}$ & \\
\hline atratus & (Olivier, 1790) & Meligethes & atratus & Rosa spp. & W Palaearctic Region, N China, Russia & MHNP & \\
\hline aurantirugosus & $\begin{array}{l}\text { Audisio, Sabatelli } \\
\text { \& Jelínek, } 2015\end{array}$ & Odonthogethes & aurantirugosus & Rubus sp?? & W Nepal & IZAS & $\begin{array}{l}\text { female } \\
\text { unknown }\end{array}$ \\
\hline aureolineatus & $\begin{array}{l}\text { Audisio, Sabatelli } \\
\text { \& Jelínek, } 2015\end{array}$ & Meligethes & $\begin{array}{l}\text { auripilis/ } \\
\text { binotatus }\end{array}$ & Rosa spp. & SW China (Sichuan) & NMPC & $\begin{array}{l}\text { female } \\
\text { genitalia } \\
\text { unknown }\end{array}$ \\
\hline auricomus & Rebmann, 1956 & Meligethes & $\begin{array}{l}\text { auripilis/ } \\
\text { binotatus }\end{array}$ & Rosa sp.? & SE China (Fujian, Jiangxi) & SMF & \\
\hline aurifer & $\begin{array}{l}\text { Audisio, Sabatelli } \\
\& \text { Jelínek, } 2015\end{array}$ & Meligethes & $\begin{array}{l}\text { auripilis/ } \\
\text { binotatus }\end{array}$ & Rosa spp. & Central China (Shaanxi, Shanxi) & NMPC & \\
\hline auripilis & Reitter, 1889 & Meligethes & $\begin{array}{l}\text { auripilis/ } \\
\text { binotatus }\end{array}$ & Rosa spp. & $\begin{array}{l}\text { SW and NW China (Sichuan, Yunnan, } \\
\text { Gansu, Shanxi, Shaanxi) }\end{array}$ & unknown & \\
\hline auropilosus & $\begin{array}{l}\text { Liu, Yang, } \\
\text { Huang, Jelínek \& } \\
\text { Audisio, } 2016\end{array}$ & Meligethes & nepalensis & Rosa spp.? & $\begin{array}{l}\text { SW and Central China (Xizang, Sichuan, } \\
\text { Hubei, Shaanxi) }\end{array}$ & IZAS & \\
\hline aurorugosus & $\begin{array}{l}\text { Liu, Yang, } \\
\text { Huang, Jelínek \& } \\
\text { Audisio, } 2016\end{array}$ & Odonthogethes & aurantirugosus & Rubus sp.? & W China (Xizang) & IZAS & \\
\hline binotatus & Grouvelle, 1894 & Meligethes & $\begin{array}{l}\text { auripilis/ } \\
\text { binotatus }\end{array}$ & Rosa spp. & $\begin{array}{l}\text { NE India, Nepal, SW China (Yunnan, } \\
\text { Sichuan), N Myanmar, Bhutan }\end{array}$ & MHNP & \\
\hline bourdilloni & Easton, 1968 & Odonthogethes & chinensis & Rubus sp.? & E Nepal & $\mathrm{BMNH}$ & \\
\hline brassicogethoides & $\begin{array}{l}\text { Audisio, Sabatelli } \\
\text { \& Jelínek, } 2015\end{array}$ & Odonthogethes & chinensis & Rubus sp.? & SW China (Yunnan) & NMPC & \\
\hline castanescens & Grouvelle, 1903 & Odonthogethes & ferrugineus & unknown & N India (Darjeeling), SW China (Yunnan) & MHNP & \\
\hline chinensis & Kirejtshuk, 1979 & Odonthogethes & chinensis & Rubus spp. & $\begin{array}{l}\text { W and Central China (Xizang, Yunnan, } \\
\text { Sichuan, Chongqing, Gansu, Shaanxi, } \\
\text { Henan, Hubei) }\end{array}$ & ZIN & \\
\hline cinereoargenteus & $\begin{array}{l}\text { Audisio, Sabatelli } \\
\text { \& Jelínek, } 2015\end{array}$ & Meligethes & $\begin{array}{l}\text { auripilis/ } \\
\text { binotatus }\end{array}$ & Rosa spp. & SW China (Sichuan) & NMPC & \\
\hline cinereus & Jelínek, 1978 & Meligethes & nepalensis & Rosa sp.? & Bhutan & $\mathrm{NHMB}$ & \\
\hline clinei & $\begin{array}{l}\text { Audisio, Sabatelli } \\
\text { \& Jelínek, } 2015\end{array}$ & Meligethes & $\begin{array}{l}\text { auripilis/ } \\
\text { binotatus }\end{array}$ & Rosa sp.? & SW China (Yunnan) & CAS & $\begin{array}{l}\text { female } \\
\text { unknown }\end{array}$ \\
\hline cyaneus & Easton, 1957 & Meligethes & atratus & Rosa sp.? & Japan & $\mathrm{BMNH}$ & \\
\hline denticulatus & (Heer, 1841) & Odonthogethes & denticulatus & Rubus spp. & W Palaearctic Region, N China, Russia & ETHZ & \\
\hline elytralis & $\begin{array}{l}\text { Audisio, Sabatelli } \\
\text { \& Jelínek, } 2015\end{array}$ & Meligethes & $\begin{array}{l}\text { auripilis/ } \\
\text { binotatus }\end{array}$ & Rosa spp. & SW China (Sichuan) & NMPC & \\
\hline ferrugineus & Reitter, 1873 & Odonthogethes & ferrugineus & unknown & N India (Sikkim) & MHNP & \\
\hline ferruginoides & $\begin{array}{l}\text { Audisio, Sabatelli } \\
\text { \& Jelínek, } 2015\end{array}$ & Odonthogethes & ferrugineus & $\begin{array}{l}\text { Pyracantha } \\
\text { sp. }\end{array}$ & Central and SW China (Hubei, Sichuan) & NMPC & \\
\hline flavicollis & Reitter, 1873 & Odonthogethes & flavicollis & $\begin{array}{l}\text { Photinia } \\
\text { sp.? }\end{array}$ & $\begin{array}{l}\text { E Russia, Japan, North Korea, SW, SE and } \\
\text { Central China (Henan, Zhejiang, } \\
\text { Chongqing, Jiangxi, Taiwan) }\end{array}$ & $\mathrm{BMNH}$ & \\
\hline flavimanus & Stephens, 1830 & Meligethes & atratus & Rosa spp. & W Palaearctic Region, N China, Russia & $\mathrm{BMNH}$ & \\
\hline griseus & Jelínek, 1978 & Meligethes & nepalensis & Rosa sp.? & Bhutan & $\mathrm{NHMB}$ & \\
\hline hammondi & Kirejtshuk, 1980 & Meligethes & atratus & Rosa spp. & $\begin{array}{l}\text { W and central China (Shaanxi, Sichuan, } \\
\text { Shanxi, Henan, Hubei) }\end{array}$ & $\mathrm{BMNH}$ & \\
\hline henan & $\begin{array}{l}\text { Audisio, Sabatelli } \\
\text { \& Jelínek, } 2015\end{array}$ & Odonthogethes & chinensis & Rubus sp.? & Central China (Henan) & NMPC & \\
\hline inexpectatus & Liu, Huang, & Odonthogethes & chinensis & Rubus sp.? & SW China (Sichuan) & NWAU & \\
\hline
\end{tabular}


Table 1 Summary of information on all 62 described species of Meligethes s.str. and Odonthogethes (Continued)

\begin{tabular}{|c|c|c|c|c|c|c|c|}
\hline specific epithet & $\begin{array}{l}\text { author(s) and } \\
\text { year of } \\
\text { description }\end{array}$ & subgenus & $\begin{array}{l}\text { species } \\
\text { group }\end{array}$ & $\begin{array}{l}\text { host- } \\
\text { plant }\end{array}$ & distribution & type(s) & notes \\
\hline & $\begin{array}{l}\text { Cline, Sabatelli \& } \\
\text { Audisio, } 2017\end{array}$ & & & & & & \\
\hline lloydi & Easton, 1968 & Odonthogethes & pectoralis & Malus sp.? & Nepal, SW China (Yunnan) & $\mathrm{BMNH}$ & \\
\hline luteomaculatus & $\begin{array}{l}\text { Liu, Huang, Cline } \\
\text { \& Audisio, } 2018\end{array}$ & Odonthogethes & chinensis & Rubus sp?? & Central China (Hubei) & NWAU & \\
\hline luteoornatus & $\begin{array}{l}\text { Audisio, Sabatelli } \\
\text { \& Jelínek, } 2015\end{array}$ & Odonthogethes & chinensis & Rubus sp.? & SW China (Yunnan) & CAS & \\
\hline lutra & Solsky, 1876 & Meligethes & vulpes & Rosa spp. & Uzbekistan & ZMUM & \\
\hline macrofemoratus & $\begin{array}{l}\text { Liu, Yang, } \\
\text { Huang, Jelínek \& } \\
\text { Audisio, } 2016\end{array}$ & Meligethes & $\begin{array}{l}\text { auripilis/ } \\
\text { binotatus }\end{array}$ & Rosa spp. & Central China (Ningxia, Shaanxi, Hubei) & MHBU & \\
\hline marmota & $\begin{array}{l}\text { Audisio, Sabatelli } \\
\text { \& Jelínek, } 2015\end{array}$ & Meligethes & $\begin{array}{l}\text { auripilis/ } \\
\text { binotatus }\end{array}$ & Rosa sp.? & Nepal & MHNG & \\
\hline martes & $\begin{array}{l}\text { Audisio, Sabatelli } \\
\text { \& Jelínek, } 2015\end{array}$ & Meligethes & vulpes & Rosa sp.? & SW and N China (Shaanxi, Shanxi, Sichuan) & NMPC & \\
\hline melleus & Grouvelle, 1908 & Meligethes & vulpes & Rosa spp. & $\begin{array}{l}\text { Myanmar, N India, N Pakistan, Afghanistan, } \\
\text { S Tajikistan, Nepal }\end{array}$ & MNHN & \\
\hline nepalensis & Easton, 1968 & Meligethes & nepalensis & Rosa spp. & Nepal, N India & $\mathrm{BMNH}$ & \\
\hline nigroaeneus & $\begin{array}{l}\text { Audisio, Sabatelli } \\
\text { \& Jelínek, } 2015\end{array}$ & Odonthogethes & chinensis & Rubus sp.? & SW China (Yunnan) & CAS & \\
\hline nivalis & $\begin{array}{l}\text { Audisio, Sabatelli } \\
\text { \& Jelínek, } 2015\end{array}$ & Meligethes & $\begin{array}{l}\text { auripilis/ } \\
\text { binotatus }\end{array}$ & Rosa sp.? & SW China (Xizang, Yunnan, Chongqing) & NMPC & \\
\hline occultus & $\begin{array}{l}\text { Audisio, Sabatelli } \\
\text { \& Jelínek, } 2015\end{array}$ & Odonthogethes & chinensis & Rubus sp.? & SW China (Yunnan) & NMPC & $\begin{array}{l}\text { male } \\
\text { unknown }\end{array}$ \\
\hline pallidoelytrorum & $\begin{array}{l}\text { Chen \& } \\
\text { Kirejtshuk, } 2013\end{array}$ & Odonthogethes & chinensis & Rubus sp. & SW China (Sichuan) & IZAS & \\
\hline pectoralis & Rebmann, 1956 & Odonthogethes & pectoralis & Malus sp. & $\begin{array}{l}\text { S Japan, SW, SE and Central China } \\
\text { (Guizhou, Hubei, Fujian, Zhejiang, Taiwan) }\end{array}$ & SMF & \\
\hline pseudochinensis & $\begin{array}{l}\text { Audisio, Sabatelli } \\
\text { \& Jelínek, } 2015\end{array}$ & Odonthogethes & chinensis & $\begin{array}{l}\text { Pyracantha } \\
\text { sp. }\end{array}$ & Central China (Chongqing, Shaanxi, Hubei) & NMPC & \\
\hline pseudopectoralis & $\begin{array}{l}\text { Audisio, Sabatelli } \\
\text { \& Jelínek, } 2015\end{array}$ & Odonthogethes & pectoralis & Malus sp.? & SW China (Yunnan, Sichuan) & NMPC & \\
\hline sadanarii & $\begin{array}{l}\text { S.-T. Hisamatsu, } \\
2009\end{array}$ & Odonthogethes & pectoralis & Malus sp.? & SE China (Taiwan) & MNST & \\
\hline schuelkei & $\begin{array}{l}\text { Audisio, Sabatelli } \\
\text { \& Jelínek, } 2015\end{array}$ & Odonthogethes & chinensis & Rubus sp.? & W China (Sichuan, Shaanxi?) & NMPC & \\
\hline scrobescens & $\begin{array}{l}\text { Chen, Lin, } \\
\text { Huang \& Yang, } \\
2015\end{array}$ & Odonthogethes & chinensis & Rubus sp.? & SW China (Sichuan, Hubei, Chongqing) & IZAS & \\
\hline semenovi & Kirejtshuk, 1979 & Meligethes & $\begin{array}{l}\text { auripilis/ } \\
\text { binotatus }\end{array}$ & Rosa spp. & $\begin{array}{l}\text { SE Russia (Ussuri), Central, SW and NW } \\
\text { China (Sichuan, Hubei, Shaanxi) }\end{array}$ & ZIN & \\
\hline shirakii & $\begin{array}{l}\text { S. Hisamatsu, } \\
1956\end{array}$ & Odonthogethes & ferrugineus & Prunus sp.? & $\begin{array}{l}\text { S Japan (Kyū-Shū), SE China (Guizhou, } \\
\text { Zhejiang, Taiwan) }\end{array}$ & EUMJ & \\
\hline simulator & $\begin{array}{l}\text { Audisio, Sabatelli } \\
\text { \& Jelínek, } 2015\end{array}$ & Odonthogethes & chinensis & Rubus sp.? & Central China (Gansu, Shaanxi) & NMPC & \\
\hline stenotarsus & $\begin{array}{l}\text { Audisio, Sabatelli } \\
\text { \& Jelínek, } 2015\end{array}$ & Meligethes & $\begin{array}{l}\text { auripilis/ } \\
\text { binotatus }\end{array}$ & Rosa sp.? & SW China (N Yunnan, Xizang) & NKMS & \\
\hline torquatus & Jelínek, 1997 & Meligethes & atratus & Rosa spp. & SE China (Taiwan) & NMPC & \\
\hline transmissus & Kirejtshuk, 1988 & Meligethes & $\begin{array}{l}\text { auripilis/ } \\
\text { binotatus }\end{array}$ & Rosa spp. & SW China (Sichuan, Yunnan) & ZIN & \\
\hline trapezithorax & Liu, Huang, Cline & Odonthogethes & chinensis & Rubus sp.? & Central China (Hubei) & NWAU & \\
\hline
\end{tabular}


Table 1 Summary of information on all 62 described species of Meligethes s.str. and Odonthogethes (Continued)

\begin{tabular}{|c|c|c|c|c|c|c|c|}
\hline specific epithet & $\begin{array}{l}\text { author(s) and } \\
\text { year of } \\
\text { description }\end{array}$ & subgenus & $\begin{array}{l}\text { species } \\
\text { group }\end{array}$ & $\begin{array}{l}\text { host- } \\
\text { plant }\end{array}$ & distribution & type(s) & notes \\
\hline tricuspidatus & $\begin{array}{l}\text { Liu, Huang, Cline } \\
\text { \& Audisio, } 2018\end{array}$ & Odonthogethes & chinensis & Rubus sp.? & Central China (Hubei) & NWAU & \\
\hline tryznai & $\begin{array}{l}\text { Audisio, Sabatelli } \\
\text { \& Jelínek, } 2015\end{array}$ & Meligethes & $\begin{array}{l}\text { auripilis/ } \\
\text { binotatus }\end{array}$ & Rosa spp. & SW China (Yunnan/ Xizang border) & NMPC & \\
\hline violaceus & Reitter, 1873 & Meligethes & atratus & Rosa spp. & $\begin{array}{l}\text { China (Anhui, Shaanxi, Hubei, Zhejiang, } \\
\text { Fujian, Guizhou, Jiangxi, Yunnan, Sichuan), } \\
\text { SE Russia (Ussuri), Japan }\end{array}$ & $\mathrm{BMNH}$ & \\
\hline volkovichi & $\begin{array}{l}\text { Audisio, Sabatelli } \\
\text { \& Jelínek, } 2015\end{array}$ & Meligethes & nepalensis & Rosa sp.? & SW China (Yunnan) & CAS & \\
\hline vulpes & Solsky, 1876 & Meligethes & vulpes & Rosa spp. & $\begin{array}{l}\text { Uzbekistan, Kyrgyzstan, Tajikistan, } \\
\text { Turkmenistan, NW China (Xinjiang) }\end{array}$ & ZMUM & \\
\hline wagneri & Rebmann, 1956 & Odonthogethes & denticulatus & $\begin{array}{l}\text { Sorbaria } \\
\text { sp.? }\end{array}$ & $\begin{array}{l}\text { SE and Central China (Fujian, Zhejiang, } \\
\text { Taiwan, E Shaanxi) }\end{array}$ & SMF & \\
\hline xenogynus & $\begin{array}{l}\text { Audisio, Sabatelli } \\
\text { \& Jelínek, } 2015\end{array}$ & Odonthogethes & ferrugineus & Rubus sp.? & SW and central China (Sichuan, Shaanxi) & NMPC & \\
\hline yak & $\begin{array}{l}\text { Liu, Yang, } \\
\text { Huang, Jelínek \& } \\
\text { Audisio, } 2016\end{array}$ & Meligethes & $\begin{array}{l}\text { auripilis/ } \\
\text { binotatus }\end{array}$ & Rosa sp.? & SW China (Sichuan) & IZAS & $\begin{array}{l}\text { female } \\
\text { unknown }\end{array}$ \\
\hline
\end{tabular}

represents the main hot-spot of the genus, ca. $85 \%$ of the known species being at least marginally distributed in this country (Fig. $1 \mathrm{~b}-\mathrm{c}$, and Table 1) [7, 10].

Species belonging to this supposed monophyletic genus are characterized by the following unequivocal autoapomorphic morphological and bionomical characters:

1) temples behind eyes (posterolateral view): with distinct, deep elliptical pit, positioned inside the posterior terminal portion of the antennal grooves (Fig. 2a); or with distinct, shallow, subcircular pit, placed more dorsad, outside antennal grooves, close to the posteriorlateral edge of the eye (Fig. 2b; pits on temples or inside antennal grooves are absent in all other Meligethinae exhibiting not raised notosternal sutures). 2) distal posterior portion of ventral antennal grooves (observed in ventral view): abruptly sloping, markedly delimited, deep, and distinctly wider than median portion (differently shaped in all other Meligethinae exhibiting not raised notosternal sutures). 3) larval development: on Rosaceae.

Several other morphological characters are shared with its supposed sister genus Brassicogethes Audisio \& Cline, 2009 (Fig. 1, a (c)) [2, 5, 24, 26], whose included species all develop as larvae on the unrelated plant family Brassicaceae. These characters include, but are not limited to: 1) terminal tarsomere simple, not toothed at base (in all Meligethes s.str.; slightly to strongly toothed at base in Odonthogethes) (Fig. 2k-m); 2) terminal maxillary palpomera long and slender, ca. $3 \times$ longer than wide (Fig. $2 \mathrm{e})$; 3) notosternal sutures usually not distinct, even in anterior portion (Fig. 2f), except in 3 species of Meligethes s.str.: $M$. violaceus, $M$. torquatus and $M$. cyaneus
(Table 1 and Tables $3-4$ in Additional files); 4) protibiae bearing only small, minute and subequal cuticular teeth along outer edge (Fig. 1 a (a), (b), (c), 2 g); 5) protibiae usually long and slender, up to 4-4.5× longer than wide (Fig. 1 a (a), (b), (c), 2 g); 6) pronotum scarcely convex, at least partially flattened at sides, with posterior angles almost right or (in most Meligethes s.str.) slightly turned posteriad (Fig. 1 a (a), (b), (c); 7) complete absence of circum-ocular furrows ("occipital sulci") when viewed dorsally (Fig. 2 (i), (j)); 8) semi-circular arched impressions on both sides of the proximal basal portion of the last abdominal ventrite large and markedly distinct (Fig. 2 (d)); 9) semi-circular arched impressions on both sides of the proximal basal portion of the pygidium arcuately and regularly convergent distad (Fig. 2 (n)); 10) male genitalia with plesiotypic tegminal shape, tegmen characterized in most species by a deep, V-shaped incision (Fig. 2 (o)-(r)), similarly exhibited by several other basal Meligethinae genera, e.g., Meligethinus Grouvelle, Micropria Grouvelle, Pria Stephens, Microporum Waterhouse, and Cryptarchopria Jelínek.

\section{Trophic relationships of Meligethes with Rosaceae host- plants}

Rosaceae is a middle-sized plant family that includes some 3000 species and a little less than 100 genera in 3 recognized subfamilies (Rosoideae, Spiraeoideae, and Dryadoideae) [35, 36]. Although exhibiting a worldwide distribution, Rosaceae are particularly diverse in northern Hemisphere temperate forests, where several genera and species of woody shrubs and small trees are important components of local forest communities. The family 
Table 2 Species and specimens of [Meligethes s.str. and Odonthogethes], Brassicogethes and Meligethinus used for molecular analysis. GenBank accession numbers for each sample and location are also reported

\begin{tabular}{|c|c|c|c|c|c|}
\hline Species & Sample ID & Localities & $\mathrm{COI}$ & $16 S$ & $C A D$ \\
\hline Meligethes auripilis & MAU1_1 & China- Sichuan, Kangding co & MT949505 & MT957159 & MT966856 \\
\hline Meligethes auripilis & MAU1_2 & China- Sichuan, Kangding co & MT949506 & MT957160 & MT966857 \\
\hline Meligethes auropilosus & MAURO1_1 & China- Hubei, Shennongjia Forest, Shennong Peak area & MT949504 & MT957158 & MT966855 \\
\hline Meligethes binotatus & MBI1_1 & China- Sichuan-Xiangcheng co. & MT949503 & MT957157 & MT966854 \\
\hline Meligethes chinensis & $4 C 2$ & China- Chongqing, Shizhu, Huangshui & MT949517 & MT957171 & MT966865 \\
\hline Meligethes elytralis & MEL1_1 & China- Sichuan, Xiangcheng co & MT949509 & MT957163 & MT966860 \\
\hline Meligethes ferruginoides & MFE1_1 & China- Sichuan-Moxi-Yanzigou & MT949511 & MT957165 & / \\
\hline Meligethes hammondi & MHA 1_1 & China- Hubei, Shennongjia forest, Muyu & MT949502 & MT957156 & MT966853 \\
\hline Meligethes luteomaculatus & MLU1_1 & China- Hubei, Shennongjia Forest, Shennong Peak area & MT949518 & MT957172 & MT966866 \\
\hline Meligethes macrofemoratus & MMA1_1 & China- Hubei, Shennongjia forest & MT949510 & MT957164 & MT966861 \\
\hline Meligethes pallidoelytrorum & MPA2_1 & China-Sichuan-Ganzi-Moxi Town & MT949521 & MT957175 & MT966869 \\
\hline Meligethes pectoralis & MPEC1_1 & China- Guizhou-Tongzi-Louguan-Mts-Wanmuhuahai & MT949515 & MT957169 & / \\
\hline Meligethes pectoralis & MPEC1_2 & China- Guizhou-Tongzi-Louguan-Mts-Wanmuhuahai & MT949514 & MT957168 & / \\
\hline Meligethes pseudochinensis & $4 \mathrm{~A} 10$ & China- Hubei, Shennongjia forest, Muyu & MT949523 & MT957177 & MT966871 \\
\hline Meligethes pseudochinensis & $4 C 5$ & China- Chongqing, Shizhu, Huangshui & MT949522 & MT957176 & MT966870 \\
\hline Meligethes semenovi & $2 C 41$ & China- Tibet, Shannan, Cuona, Gongri & MT949508 & MT957162 & MT966859 \\
\hline Meligethes semenovi & $2 C 42$ & China- Tibet, Shannan, Cuona, Gongri & MT949507 & MT957161 & MT966858 \\
\hline Meligethes scrobescens & $4 \mathrm{~B} 7$ & China- Chongqing, Shizhu, Huangshui & MT949516 & MT957170 & MT966864 \\
\hline Meligethes transmissus & MTR1_1 & China- Sichuan, Kangding co & MT949501 & MT957155 & MT966852 \\
\hline Meligethes violaceus & MVI1_1 & China- Zhejiang-Quzhou City-Jiangshan City & MT949519 & MT957173 & MT966867 \\
\hline Meligethes wagneri & $4 C 4$ & China- Chongqing, Shizhu, Huangshui & MT949520 & MT957174 & MT966868 \\
\hline Meligethes xenogynus & $3 \mathrm{~A} 31$ & China- Shaanxi, Meixian, Haoping temple & MT949512 & MT957166 & MT966862 \\
\hline Meligethes xenogynus & $3 \mathrm{~A} 32$ & China-Shaanxi, Meixian, Haoping temple & MT949513 & MT957167 & MT966863 \\
\hline Brassicogethes coracinus & CR8_1 & Turkey-Ardahan-road between Göle and Susuz & MT949498 & MT957152 & MT966849 \\
\hline Brassicogethes aeneus & BAE13_3 & Italy- Lazio- Pomezia - Borgo di pratica di mare & MT949496 & MT957150 & MT966847 \\
\hline Brassicogethes aeneus & BAE13_4 & Italy- Lazio- Pomezia - Borgo di pratica di mare & MT949497 & MT957151 & МT966848 \\
\hline Brassicogethes salvan & BSA1.1 & Italy- Piemonte- Mt.Palafrè- Lago inf. Del Frisson & MT949495 & MT957149 & MT966846 \\
\hline Meligethinus peringueyi & MEP1_1 & Mozambique- Maputo,Reserva Especial & MT949500 & MT957154 & MT966851 \\
\hline Meligethinus dolosus & MED1_2 & Mozambique- Maputo,Reserva Especial & MT949499 & MT957153 & MT966850 \\
\hline
\end{tabular}

is peculiar in producing several different and highly distinctive types of fruits, including economically important edible fruits such as apples, pears, peaches, apricots, prunes, strawberries, cherries, raspberries, and blackberries. Meligethes s.l. (i.e., Meligethes s.str. + Odonthogethes) specialize on several genera and species of Rosaceae, although, differently from the majority of other Meligethinae lineages [2,37, 38], only large shrubs and small trees of the two main subfamilies Rosoideae and Spiraeoideae are utilized [10]. No Meligethes (nor other Meligethinae genera) are, in fact, known to develop as larvae on herbaceous Rosaceae (e.g. the widespread and species-rich Potentilla, Fragaria, and Geum), despite species of these same plant genera being commonly used by adults of several different genera and species of Meligethinae as occasional food-plants [1].
Despite limited or incomplete information on the larval host-plants of some species, in general Meligethes s.l. appear to be associated with Rosaceae belonging to Rosa, Rubus, Malus, Prunus, Crataegus, Pyracantha, Sorbaria, and Photynia $[1,7,10]$. A couple of these (Rosa, Rubus) belong to the subfamily Rosoideae [35], whereas others (Malus, Prunus, Crataegus, Pyracantha, Sorbaria, and Photynia) to the subfamily Spiraeoideae. The two most commonly used larval hosts of Meligethes s.l. are Rosa L. and Rubus L. Rosa, which represents the only known larval host of species in the subgenus Meligethes s.str., includes some 200 species worldwide; Rubus, which represents the main larval hosts of species in the purported subgenus Odonthogethes, includes at least between 400 and 1000 species [35, 39-41]. Both genera exhibit biodiversity hot-spots in China and neighboring 


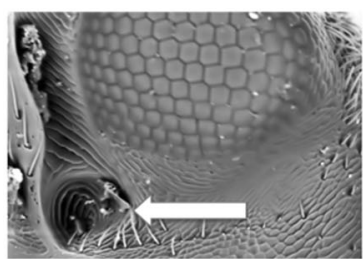

(a)
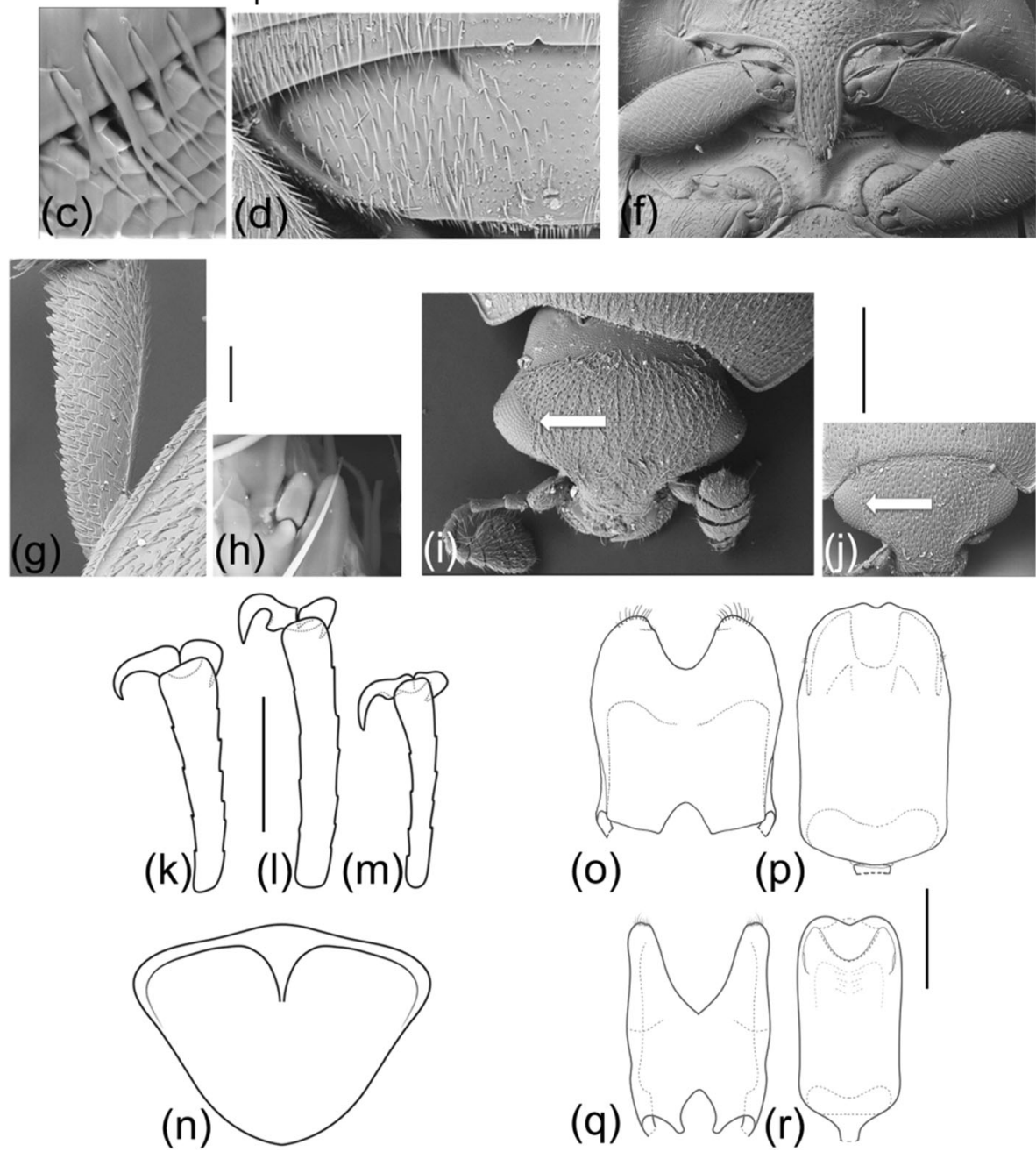

(0)
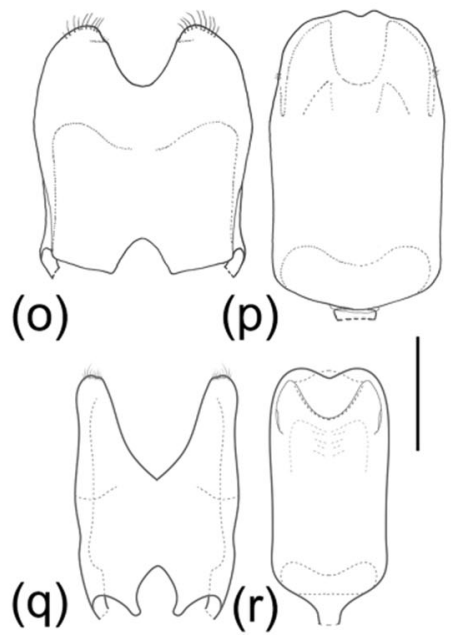

Fig. 2 SEM pictures of Meligethes (s.l.) spp.; a left eye and ventral portion of temples (Meligethes flavimanus); b left eye and lateral portion of temples (Odonthogethes castanescens). Arrows indicate shared pit in ventral subocular lateral portions of temples (inside terminal posterior portion of ventral furrows in members of subgenus Meligethes s. str., laterad and more dorsad in members of Odonthogethes). c Microsetae along posterior edge of pronotum of Odonthogethes castanescens. $\mathbf{d}$ Left portion of last visible abdominal ventrite of Odonthogethes denticulatus. e Right maxillary palp and labial palp of Odonthogethes denticulatus. $\mathbf{f}$ Prosternum, prosternal process and mesoventrite of Odonthogethes denticulatus. $\mathbf{g}$ Left protibia, dorsal view, of Meligethes atratus. h Close-up of left protibia, dorsal view, of Odonthogethes castanescens, with predistal tooth and spicule. Dorsal head view in Meligethinae; i Meligethes atratus; $\mathbf{j}$ Brassicogethes aeneus. Arrows of Figs (i) and (j) point to the shared absence of dorsal circumocular furrows on dorsal head surface. Drawings of Meligethes (s. l.) spp. (k-r); terminal left metatarsomeres: $\mathbf{k}$ Meligethes atratus; I Odonthogethes denticulatus; $\mathbf{m}$ Odonthogethes flavicollis. $\mathbf{n}$ Last visible dorsal abdominal segment (pygidium) of Odonthogethes flavicollis. Male genitalia (tegmen and median lobe of aedeagus) in Meligethes (s. I.) and Brassicogethes; o, p Meligethes (M.) atratus; q, $\mathbf{r}$ Brassicogethes salvan (Audisio, Antonini \& De Biase, 2003). Scale bar $=0.01 \mathrm{~mm}$ (Fig. h); $=0.02 \mathrm{~mm}$ (Fig. C); $0.1 \mathrm{~mm}$ (Figs e, g, k-m); $=0.12 \mathrm{~mm}$ (Figs a, b) $;=0.18 \mathrm{~mm}$ (Fig. d) $;=0.2 \mathrm{~mm}$ (Figs o-r); $=0.4 \mathrm{~mm}$ (Figs i, j); $=0.5 \mathrm{~mm}$ (Figs f, $\mathrm{n}$ ) 
areas, where ca. 100 and more than 200 species respectively are known to occur [42]. A similar pattern occurs in Meligethes s.l., and in its constituent subgenera, species-groups and complexes $[1,2,7]$. Due to these above mentioned botanical phylogenetic and biogeographic scenarios, we comprehensively explored the evolutionary trajectories within Meligethes s.l. also to provide some hints on the role of the relationships between this group of pollen beetles and their larval host plants.

\section{Results}

\section{Morphological phylogeny and cladistic analysis}

The cladistic analysis of the matrix under implied weights $(k 10.72)$ yielded 429 equally most parsimonious trees with a total length of 184 steps, a consistency index (C.I.) of 0.45 and a retention index (R.I) of 0.87 (Fig. 3).

Meligethes s. l. and Brassicogethes were recovered as sister taxa as they shared 1 nonhomoplasious apomorphy (48:0, axillary triangular spaces close to proximal base at both sides of first abdominal ventrite larger than metatrochanter). The monophyly of Meligethes s.l. relied on 1 non homoplasious (12:1, postocular temples with pit) and 1 homoplasious (61:0) apomorphies. In turn, Meligethes included two main subclades: Meligethes s. str. (Clade A) and Odonthogethes (Clade B) (Fig. 3). Meligethes s. str. Was supported as monophyletic based on 3 homoplasious apomorphies $(24: 2 ; 36: 2 ; 64: 1)$. The monophyly of Odonthogethes was based on 5 nonhomoplasious (19:2, 3rd and 2nd antennomeres (ratio L03)/ L02J) $<=0.9 ; 21: 2$, antennal club $<1.10-1.15$ times as long as wide; 39:0, posterior edge of metaventrite outer posterior angles well distinct; 44:1, tarsal claws distinctly toothed at base; 54:1, longest setae on distal portion of parameres in dorsal view (ratio THLE/LETE) $=>0.14$ ) and 3 homoplasious $(16: 1 ; 22: 0 ; 34: 0)$ apomorphies. The sister-group relationship between Meligethes s. 1. and Brassicogethes, as well as the occurrence of the two main subclades within Meligethes s. 1., i.e. Meligethes s. str. and Odonthogethes, was also confirmed by ML analysis of morphological data (Additional file 8).

\section{Molecular phylogeny and divergence time estimation}

Our final dataset consisted of 1841 bp (COI: 578 bp, 16S: 491 bp, CAD: 772) obtained from 29 specimens (see Table 2). Phylogenetic trees resulting from the BI and ML analyses showed congruent topologies (Fig. 4); only BI posterior probability values and ML bootstrap values exceeding $70 \%$ are shown as BI/ML. Our phylogram indicated the presence of two highly supported principal clades, corresponding to the Meligethes s.str. Species $(\mathrm{BI}=1 / \mathrm{ML}=100)$ and two clades including Odonthogethes and Brassicogethes species, respectively $(\mathrm{BI}=1$ / $\mathrm{ML}$ =92). Moreover, the monophyly and the sister- group relationships of a clade including Meligethes s.str. + [Odonthogethes + Brassicogethes] was well supported in both BI (0.85) and ML (81). The trees obtained from single-gene alignments are reported in Additional files $(4,5$ and 6$)$.

Divergence time estimates from BEAST are depicted in Fig. 5. With a calibration of 0.0126 substitutions/site per My, the possible origin of the core members of the Meligethes complex of genera [Meligethes s.str. + Odonthogethes + Brassicogethes] (i.e., divergence from Meligethinus) was estimated to be approximately in the Middle Miocene, ca. 15-14 Mya. The split of Odonthogethes from Meligethes can be traced back to the Middle Miocene (12.27 Mya; 95\% HPD: 9.10-15.30 Mya) and that of Brassicogethes from Odonthogethes in the Late Miocene (11.15 Mya; 95\% HPD: 8.20-14.10 Mya).

\section{Molecular vs. morphological analyses}

Evidence from our molecular analyses resulted in a rather good agreement with previously established morphology-based systematics of Meligethes and allied genera. Our molecular analyses suggested (Figs. 4-5) that most Odonthogethes species likely differentiated within the last 10 million years. These were mostly included in the $O$. chinensis species-group, the largest one within this genus, which are associated as larvae with Rubus spp. (Rosoideae) and with several other Spiraeoideae. Although the taxon sampling in our molecular analysis is rather partial, a preliminary conclusions and comparison with morphological-based results, allows confirming the monophyly of the present-day Odonthogethes. Nevertheless, the monophyly of the (sub) genus Meligethes s. str. Remains slightly ambiguous morphologically, suggesting that this taxon might be an assemblage from which Odonthogethes was derived. A future work with additional material of representatives of the $M$. vulpes species-group (Fig. 3; Meligethes vulpes and allied species, mostly from hardly accessible countries in Middle Asia) is needed to verify, through molecular data, the phylogenetic position of this isolated group within Meligethes s. str. Additionally, our molecular analysis recovered Brassicogethes as deeply nested within Meligethes pointing to a paraphyletic condition of the genus Meligethes, as recently conceived. This evidence is partially in contrast with morphological analyses, which, on the contrary, suggests a sister-group relationship between Brassicogethes and (a monophyletic) Meligethes [Meligethes s.str. + Odonthogethes] (the tree obtained from combined molecular and morphological dataset is reported in Additional file 7). In presence of this contrasting information, the hypotheses to either downgrade Brassicogethes to the subgenus level, within the genus Meligethes s. l., or, alternatively, to raise Odonthogethes to the genus rank should be taken into account. 


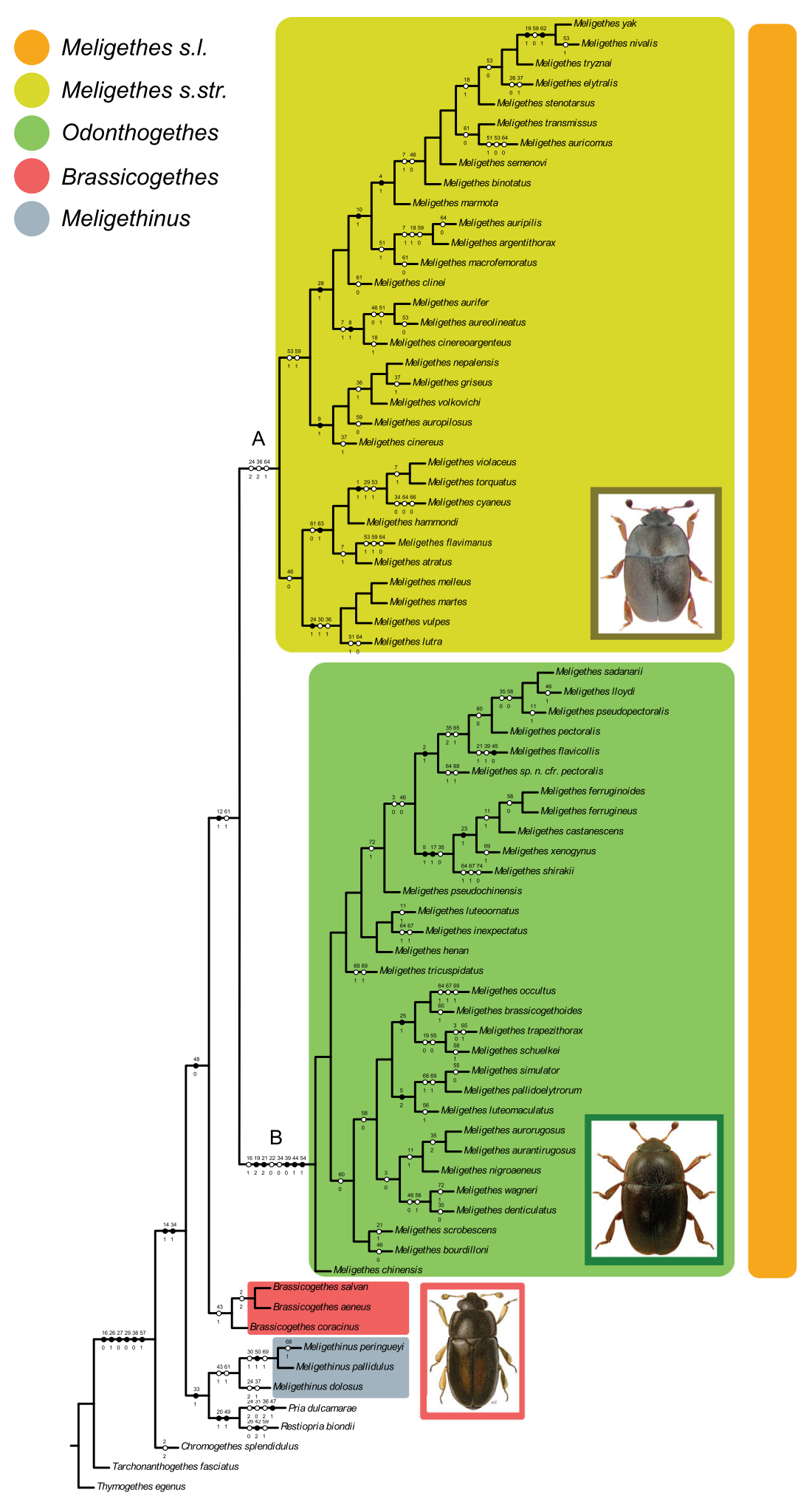

Fig. 3 (See legend on next page.) 
(See figure on previous page.)

Fig. 3 One of the most parsimonious trees based on morphological data (tree length $=184$, C.I. $=0.45$, R.I. $=0.87$ ). Homoplasious and nonhomoplasious character states are indicated with black and empty squares, respectively. Capital letters refer to clades as determined by morphological cladistic analysis (see Results and Discussion); the present-day subgeneric classification is superimposed on the $\mathrm{C}$ (Odonthogethes) and A $+B$ (Meligethes s.str.) clades. Reconstruction is based on 74 morphological and ecological characters (169 states) for 63 members of Meligethes, + Brassicogethes aeneus, B. salvan, B. coracinus, Meligethinus pallidulus, M. peringueyi, M. dolosus, Pria dulcamarae, Restiopria biondii, Tarchonanthogethes fasciatus, Chromogethes splendidulus, and Thymogethes egenus (outgroups) (see Additional files Tables 3 and 4, for character list and matrix)

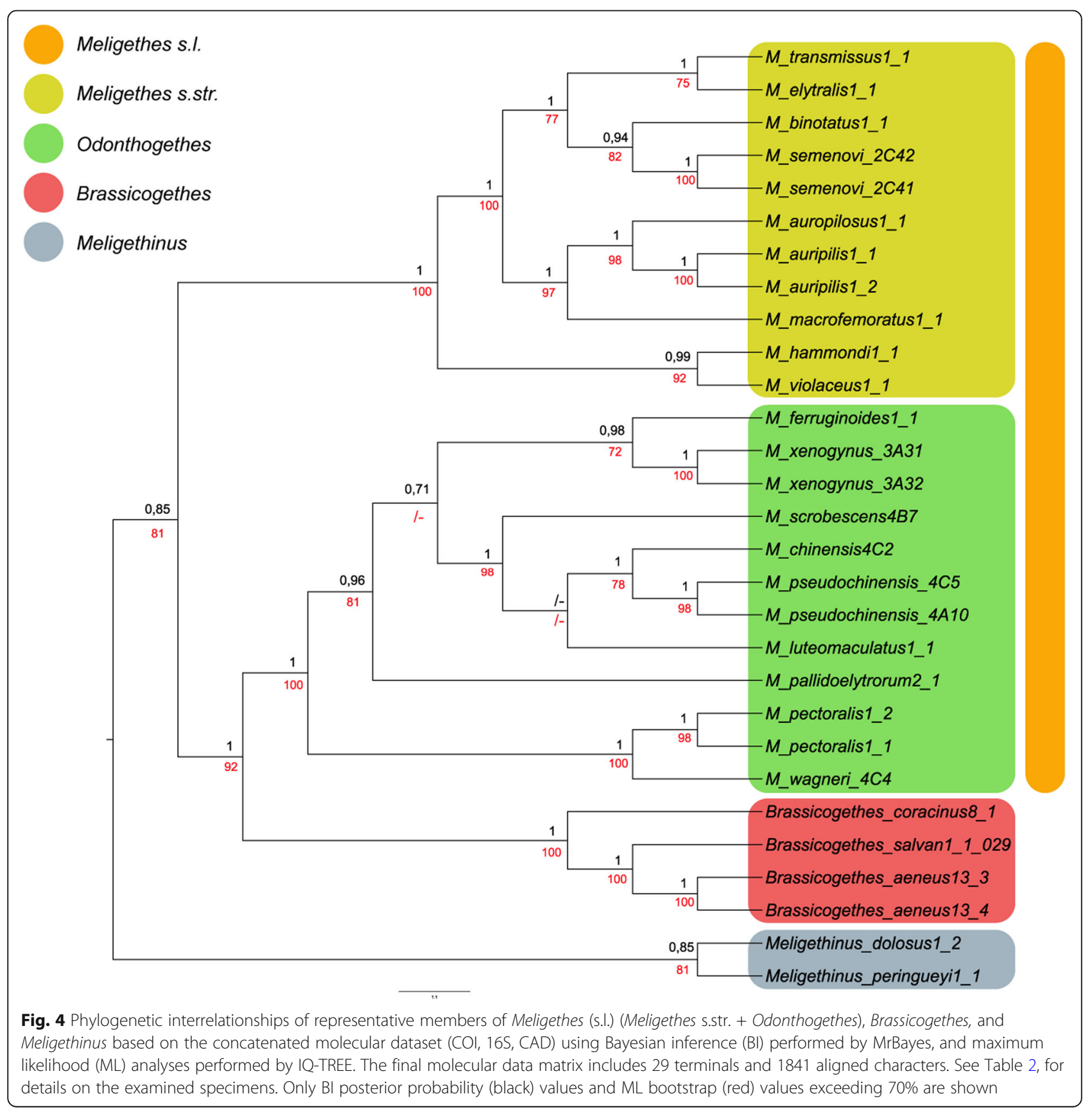




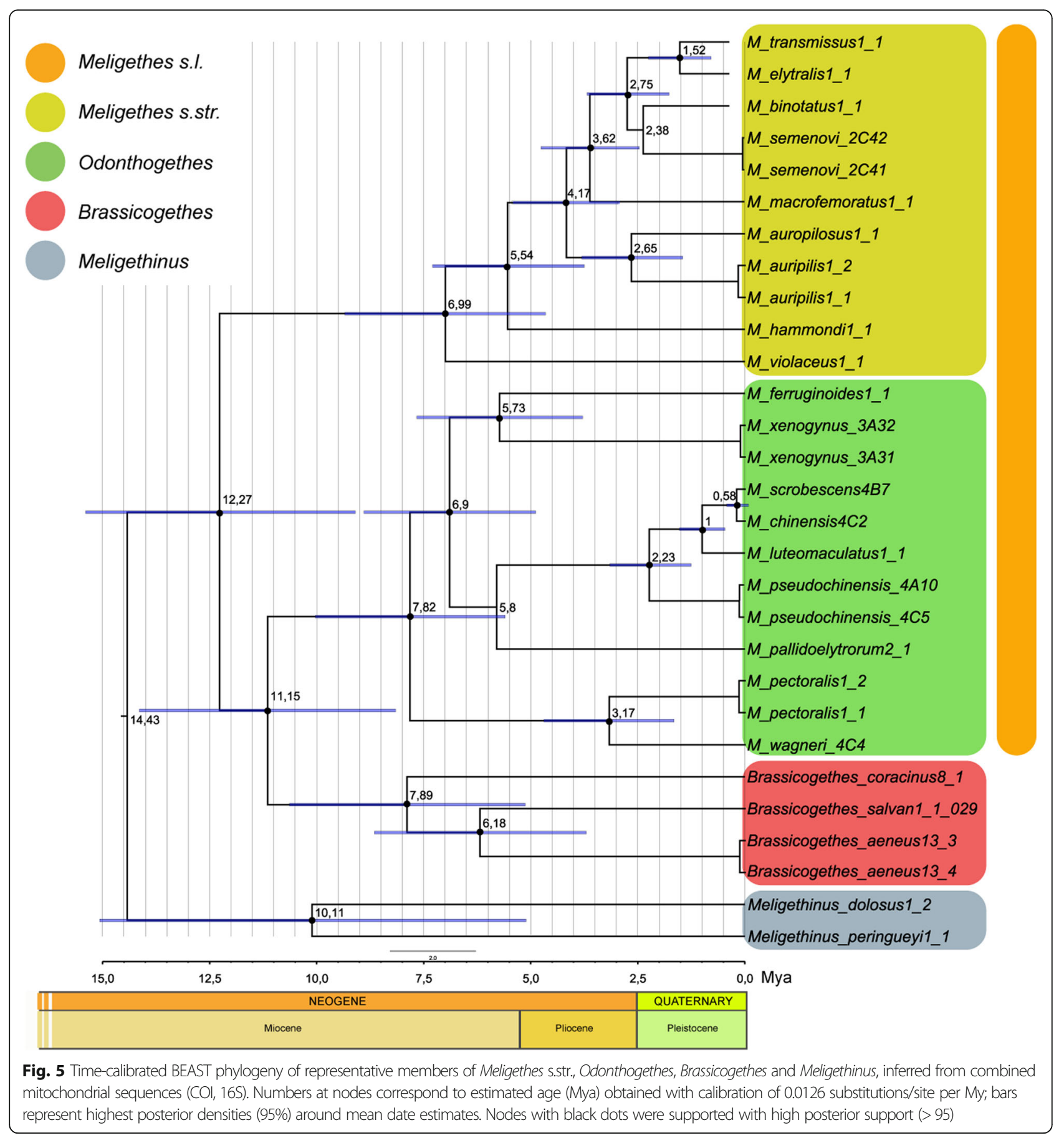

\section{Ancestral state parsimony reconstruction of larval-host- plant associations}

The ancestral state reconstruction plotted on the topology resulting from the combined morphological and molecular dataset analysis (Fig. 6) suggested that Rosaceae could be regarded as the ancestral host-plant family of Meligethes (s.l.) and Brassicogethes, although the actual succession of family-level larval shifts among the whole clade remains difficult to define.
Noteworthy, Meligethinus species are all associated with monocots in the family Arecaceae [1, 2, 43]. According to our molecular data, the common ancestor of Meligethes s. 1. subsequently colonized a new niche, probably exploiting Rosaceae in the Eastern Palaearctic, while Brassicogethes, maybe also originated in the same areas, later radiated on Brassicaceae, mostly in W Palaearctic subregion. This scenario is supported by the ancestral state reconstruction, which suggested that the 


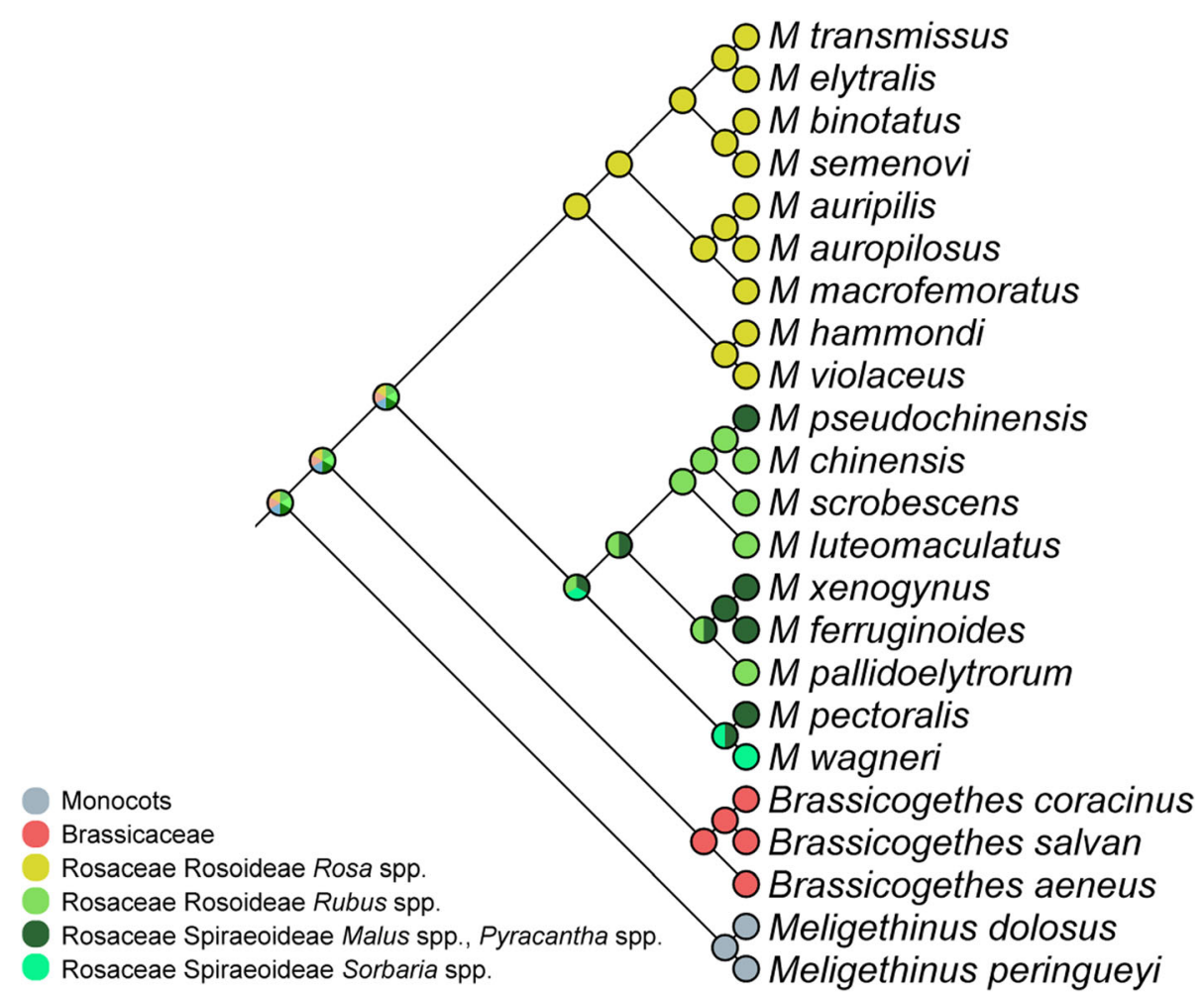

Fig. 6 The strict consensus tree based on combined morphological and molecular dataset constructed via MrBayes, superimposed on ancestral state parsimony reconstruction of larval-host-plant associations executed in MESQUITE (tribes within Rosaceae according to [35, 36, 39-41])

diverse genus Rosa L. most likely represented the ancestral host of Meligethes s. str. The larvae of most species of Meligethes s. str. Specialized on species belonging to this plant group, while members of Odonthogethes rather shifted on other Rosoideae, specifically on the genus Rubus L. Subsequently, a species of this subgenus probably colonized Rosaceae belonging to the subfamily Spiraeoideae and this ancestral host shift event may have led to a subsequent diversification of a group whose members radiated by exploiting several plants of this subfamily (e.g. Malus, Crataegus, Pyracantha and Photinia) (Fig. 6). A few isolated species belonging to both subgenera, Meligethes ( $M$. atratus, M. flavimanus, and M. torquatus) and Odonthogethes (O. shirakii) also independently colonized Spiraeoideae of the genus Prunus [1, 13]. An even more isolated host shift event was likely experienced by Odonthogethes wagneri, the only species feeding on Spiraeoideae of the genus Sorbaria, and the isolated O. flavicollis, which is the only species apparently feeding on Spiraeoideae of the genus Photinia [10]. Molecular data suggest that a more drastic ecological shift finally involved some ancestral species of Odonthogethes, able to transfer to Brassicaceae, and very likely originating the Brassicogethes clade.

\section{Discussion}

Meligethes originated in the SE Palaearctic and NE Asia and adapted to flowers and large inflorescences of Rosaceae for larval feeding. The genus was likely derived from an ancient stem of Meligethinae not distantly related to Meligethinus, Micropria, Kabakovia, Pria, and allied genera, several species of which (except among Pria and Micropria) are associated as larvae with male inflorescences of palms (Arecaceae) [1, 2, 24, 26, 43].

As for the timing of lineage splitting, the few fossil data available for Meligethinae [44, 45] are based on Baltic amber material (traditionally dated back to some 3638 Mya). However, although certainly to be referred to genera other than Meligethes as presently circumscribed, the correct classification of these fossils is still pending. The Baltic amber material from Oligocene (ca. 40-50 Mya; "Melipriopsis rasnitzyni") [45], is, for instance, hardly referable to Meligethinae, due to some characters (e.g. the distinctly bordered posterior base of pronotum; the angulose "axyllary line" on metaventrite; the ciliate pronotal sides) which are unknown among true members of this subfamily. Due to the above-mentioned uncertainties, we were not able to rely on distant external calibration points [46]. It is worth remarking, however, that even if distantly related fossils were available; these would have improperly timed the evolution of the focal 
group due to its intrinsic biological properties [47]. In fact, Meligethinae are known to be affected by rate variation among lineages, incomplete haplotype sorting and a host-plant dependent and fluctuating demography [22, 23]. Hence, we decided to tentatively provide a temporal framework for the speciation events of the target taxa by relying on the most accurate substitution rates currently available for beetles and setting a relaxed clock model to overcome some of the recognized limits of this dating method [46, 47]. Our molecular analyses suggest that timing of separation between [Meligethes + [Odonthogethes + Brassicogethes]] occurred in a time frame of ca. 11-13 Mya, whereas separation of this clade from the related genus Meligethinus probably dates back to 14-15 Mya. Therefore, the Middle Miocene (Langhian Age) could represent a plausible timing for the first Meligethes lineages to specialize on Rosaceae host plants. Meligethes later continued to evolve and adapt in Eastern Palaearctic and E Asiatic areas on Rosaceae Rosoideae, from which Odonthogethes plausibly radiated during the Late Miocene, Pliocene and Pleistocene on Spiraeoideae (Rosaceae subfamily). Brassicogethes, following their shift to Brassicaceae - likely from a stem of ancestral Odonthogethes - expanded and strongly diversified its constituent lineages mostly in W Palaearctic, paralleling a westward evolution, diversification and expansive radiation of Brassicaceae host-plants. Several closely related species of this (sub) genus likely differentiated only in the last 2 Mys [14, 22, 23]. These species, in fact, usually exhibit low levels of molecular interspecific differentiation, typical of recently specialized lineages among which genetic differentiation can be markedly slower than diversification among morphological and ecological adaptive traits. This phenomenon, well-known in different groups of recently speciated phytophagous insects [23], is probably due to the retention of common ancestral haplotypes when genetic differentiation has not had the necessary time to be fixed after speciation [23]. Among Odonthogethes, a similar phenomenon certainly involves most members of the Odonthogethes chinensis group, which, although strongly differentiated from one another based on morphological and ecological traits, exhibit low levels of interspecific genetic differentiation (Fig. 5) [Audisio et al. unpublished data].

Only a few Meligethes of the (sub) genera Meligethes and Odonthogethes were able to reach the W Palaearctic, likely during the most recent Pleistocene Glaciation $\mathrm{Cy}$ cles, maybe due to the moderate number of potential larval host-plants (indigenous Rosaceae) occurring west of Middle Asia. Mountain systems of the SE Palaearctic (in particular those in Central and S China, Nepal, Bhutan, and NE India) and transitional areas between $\mathrm{E}$ Palaearctic and N Oriental Regions, seem to represent the most active centers of speciation and evolution of
Meligethes and Odonthogethes, reflecting the local strong generic- and species-level diversification of Rosaceae [35, 36, 40-42].

As mentioned above, Meligethes s. str. Appear to be more strictly specialized on Rosa spp., while Odonthogethes appear to have adapted to a much wider range of Rosaceae genera, including: Rubus to Prunus, Sorbaria and related taxa, in the two main Rosaceae subfamilies, Rosoideae and Spiraeoideae [35, 36, 48]. The only known more highly polyphagous Meligethes s.str. Are represented by two species ( $M$. atratus and M. flavimanus) that are widespread from $\mathrm{N}$ Asia to the Iberian Peninsula. Both species are able to develop, at least in W Palaearctic, on different genera of Rosoideae and Spiraeoideae [1,7]. This could be interpreted as a recent local widening of ecological (host-plant) ranges, following post-glacial colonization of W Europe, in a region where other competing and more specialized Rosaceaedependent species of the same genus are absent.

The two largest genera of Rosaceae Rosoideae, Rubus and Rosa, split more than $40 \mathrm{Mya}$, well before the here estimated divergence between Odonthogethes (mostly associated with Rubus spp.) and Meligethes s. str. (all likely associated with Rosa spp.) that is tentatively dated ca. 12-13 Mya. This asynchronous timing clearly does not allow supporting the hypothesis of a direct coevolution [49] among these beetles and their host plants. However, a model of 'sequential evolution' - i.e. the shift of insect herbivores onto a pre-existing group of plant species [50] - could rather be raised to explain the origin of the main (deepest) specialized-clades by some ancient common ancestors able to colonize novel plant-groups of Rosoideae (Figs. 5-6). Yet, it is worth noticing that most strictly endemic and locally distributed Meligethes and Odonthogethes species from China and neighboring countries are typically associated as larvae with common and widespread floral hosts, and not to endemic plant species [10]. Therefore, geographic isolation in more or less remote mountain valleys seems to have played a more relevant role than ecological specialization to host plants in driving speciation among members included in both Meligethes and Odonthogethes clades. It is interesting to point out that Meligethes and Odonthogethes probably experienced different selective pressures respect to the W Palaearctic Brassicogethes (associated with the unrelated family Brassicaceae), whose more recently-speciated taxa were able to frequently colonize and adapt to rare and/or endemic plant species $[1,5$, 14-23].

In line with the above argument, we observed that morphology allowed to set apart and identify the main lineages of both Meligethes s.str. and Odonthogethes, although the extensive homoplasy between these groups prevented to fully resolve the phylogenetic relationships 
among species-groups (Fig. 3, Additional file 8). Additionally, several characters of both taxa, although diagnostic at the interspecific level, were widely affected by homoplasy. The sharing of so many morphological traits could suggest that members of several species-group in Meligethes s.str. and Odonthogethes, probably originated recently (e.g., in allopatry) without experiencing the strong selective pressures posed by the adaptation to numerous larval host plants and various ecological contexts, which, on the contrary, rather favored a (relatively) more rapid diversification of Brassicogethes. Consistently, in Brassicogethes, where combined phenomena of speciation triggered by larval host-plant specialization (following repeated larval host-shifts) occurred much more frequently, phylogenetic relationships among the main species-groups appear to be more clearly resolved by morphological data alone, though relationships within some morphologically recognized species groups required the support of molecular data as well [23].

We are confident that further analyses could provide more detailed phylogenetic evidence on the three involved lineages, in a more coherent scenario of their actual evolutionary relationships, despite currently partly contrasting phylogenetic information provided by morphological data and molecular markers. Additionally, molecular data suggests a markedly isolated phylogenetic position for Meligethinus dolosus Grouvelle, 1919 (from NE South Africa and Mozambique). This evidence is confirmed by morphological data [43]. This problem, as well as the internal phylogeny of Meligethinus, is beyond the scope of the present paper, and will be discussed in an upcoming effort that is specifically devoted to a morphological and molecular phylogeny of this genus [43].

\section{Conclusions}

The present study analysed 63 species of Meligethes (s.l.), setting up the basis for further research on this taxon. Judging from the relatively high number of new species (>12) discovered during less than five years of field and Museum research following the recent revision of this group [7], combined with the vast extension of scarcely explored E Palaearctic and N Asian areas (eastern Middle Asia, Northern Indian subcontinent, S China, and northern Indochina), there is a high likelihood that the actual number of species in the (sub) genera Meligethes and Odonthogethes might increase significantly to even more than 70-80 species as a whole. The species of both clades seem to be concentrated in central and southern China, specifically in subtropical evergreen broadleaf forest zones, in eastern portions of the Qinghai-Xizang Plateau alpine vegetation zone, and in southern portions of the warm temperate deciduousbroadleaf forest zone [51] (http://www.chinamaps.org/ china/china-land-cover-map-large-2.html). However, scarcely explored mountain areas of S China still possess new endemic species. These areas represent true biodiversity hot-spots (Fig. $1 \mathrm{~b}-\mathrm{c}$ ) for both Meligethes and Odonthogethes and their Rosaceae host-plants, and also likely include Nepal, Bhutan, Taiwan, and the nearly unexplored surrounding mountain areas of eastern India (Arunachal Pradesh) and northern Myanmar [7-10].

Despite the incomplete taxonomic and biogeographic coverage, the present morpho-ecological phylogenetic analysis and the thus far available molecular data seem to support at least the following conclusions:

1) Based on molecular evidence, the Meligethes complex of genera univocally represents a monophyletic lineage, including almost certainly three species-rich genera or subgenera (Meligethes s.str., Odonthogethes, and Brassicogethes), which are comparable in the number of inclusive species (32, 31 and 42 species, respectively). Other preliminary molecular data using mitochondrial and nuclear markers (COI, NADH, ITS2, PEPCK) [24, 26], suggested that the genus Meligethinus Grouvelle (a small, mostly Oriental and Afrotropical genus including some 20 species associated as larvae with male inflorescences of Arecaceae [1, 43]) is placed as a sister taxon of this clade. In fact, the phylogenetic position of Meligethes and Odonthogethes is certainly closer to both Brassicogethes and Meligethinus, than to any other recognized genera inside Meligethinae [43]. Indeed, the positional homologous insert at the apical portion of the ITS2 domain B, which, as previously demonstrated [24], is shared across all Meligethes, Odonthogethes, Brassicogethes and Meligethinus, should be considered a diagnostic sequence insertion. This combined with other slippage-derived signature sequences identified in the same paper for these related Meligethinae genera strongly corroborates a common origin of these four taxa.

2) Based only on morphological and ecological evidence (Fig. 3), the clade [Meligethes s.str. + Odonthogethes] could represent the sister-group of the genus Brassicogethes, with Meligethinus sister of this triplet of taxa. However, the molecular data presented here (Figs. 4-5) strongly suggest a different and likely more compelling scenario, with Brassicogethes and Odonthogethes being sister taxa, Meligethes being sister of the latter clade, and Meligethinus sister of this triplet of taxa.

3) In an ecological-evolutionary perspective, differently from Brassicogethes, geographic isolation in remote mountain valleys of the Eastern Palaearctic seems to have played a more relevant role than ecological specialization in species splitting and diversification within both Meligethes and Odonthogethes genera.

Finally, a short series of very recently published or upcoming papers of our research team is aimed to introduce and formalize an updated taxonomic rank for 
Meligethes, Odonthogethes, and Brassicogethes, and to describe some recently discovered new Chinese species, as well as the previously unknown males of a few additional taxa [52].

\section{Methods}

Field research

All species were collected as adults on their known or putative host-plants. Attempts were also made to obtain larvae to confirm the insect-host plant relationships. Larvae were collected alive by hand using entomological forceps inside flower buds, where they mainly develop during the early flowering season of hosts. Adults destined for morphological analyses were killed in small vials containing cork powder and a few drops of ethylacetate. Additional conspecific adults destined to molecular analyses were killed and preserved in absolute ethanol. Larvae destined for morphological analyses were killed and preserved in a mixture of $75 \%$ ethanol and $25 \%$ filtered pure water, while additional conspecific specimens destined for molecular analyses were killed and preserved in absolute ethanol. A few larvae, when available in number, destined to SEM analyses, were killed in absolute acetone to preserve the larval cuticular surface and corresponding setae, tubercles, and other projections.

In most cases, adult specimens were collected after direct observations on target plants, with some specimens of possible target beetles directly collected using an aspirator or by hand. This technique had to be employed to avoid physical damage to rare and locally protected plants via netting or beating techniques. Netting or beating were used only for locally abundant hostplants, such as widespread or even invasive species of the Rubus or Prunus in shrubby habitats. Pollen beetles on these plants are usually highly dispersed and therefore difficult to find and collect using visual observation only.

\section{Morphological phylogeny and host plant associations}

A matrix containing 69 morphological and 5 ecological characters (Tables 3-4) was compiled for all known 63 species of Meligethes. The genera Thymogethes Audisio and Cline, 2009 (represented here by T. egenus from S Europe), Brassicogethes Audisio and Cline, 2009 (B. aeneus, B., salvan and B. coracinus from S Europe), Tarchonanthogethes Audisio and Cline, 2009 (T. fasciatus from S Africa), Chromogethes Kirejtshuk, 1989 (C. splendidulus from $\mathrm{S}$ Africa), Restiopria Audisio, Jelínek and Cline, 2011 in [25] (R. biondii from S Africa), Pria Stephens 1830 (P. dulcamarae from S Europe) and Meligethinus Grouvelle, 1906 (M. pallidulus from W Mediterranean areas, and $M$. peringueyi and $M$. dolosus from Southern Africa) were selected as outgroups based on their different levels of affinities with Meligethes Stephens $[5,6,18]$. Among the 69 selected morphoecological characters, some usually significant in Meligethinae interspecific diagnostics (e.g., some from male and female genitalia) were not included. Those traits, upon cladistics analysis, resulted manifestly homoplasious in different unrelated clades, and therefore were deemed scarcely informative or even confusing in a cladistic framework. The morphological matrix was produced with MESQUITE version 3.51 [52] and subsequently analyzed in TNT version 1.5 [53]. Multistate characters were treated as unordered and zerolength branches were collapsed. Analyses were run as implicit enumeration under implied weights (concavity factor of 1 and higher), under the "traditional search option". The following parameters were applied: general RAM of $1 \mathrm{~GB}$, memory set to hold 1,000,000 trees, setting 1000 replicates with tree bisection-reconnection branch swapping and saving 1000 trees per replicate. The most fitting concavity $k$-value of the weighting function was found using the TNT script "setk.run" [53], obtaining a $k$ value 10.72 . Characters were mapped on one of the most parsimonious trees using Winclada version 1.00.08 [54]. To confirm the deepest relationships inferred by the MP analysis, we also generated a Maximum Likelihood (ML) tree using default settings in IQTREE [55].

An ancestral state parsimony reconstruction of larvalhost-plant associations was carried out in MESQUITE version 3.51 [56] using the most recent phylogeny-based general classification schemes of Rosaceae [35, 36]. The reconstruction of ancestral state parsimony for larvalhost-plant association (Fig. 6) was carried out and superimposed on our combined morphological and molecular dataset-based cladogram following the methods discussed elsewhere [23].

\section{Insect-host plant relationships and phenology}

We collected field information on life history, larval host-plants and phenology on ca. 45 out of 63 species of the genus; for some poorly known and rare species from $\mathrm{N}$ Indian subcontinent, Middle Asia, China and Japan no additional information on biology was provided. Among the analyzed species, steno-oligophagy (i.e. dependence of a single beetle species on a short series of closely related plant species belonging to the same genus or to closely related genera) is the dominant condition, while monophagous species are few.

Most species of the genus are active during the breeding season in Summer (i.e., mid-June to late July; 36 species out of 45 , i.e., $80 \%$ ), while only a couple of mostly W Palaearctic species are Spring specialists (breeding from early April to middle May; 2 species out of 45, i.e., 9\%: M. atratus and M. flavimanus). The remaining 7 
analyzed species more widely extend their annual phenology between May and August.

\section{Molecular methods \\ DNA extraction, amplification and sequencing}

A total of 29 adult specimens from 18 different Meligethes s.l. species, as well as 3 species of Brassicogethes and 2 of Meligethinus were collected alive in the field and directly killed and preserved in absolute ethanol. In Table 2, the geographic details for the species are listed. Species identifications were made using morphological characters detailed elsewhere [1,7-9] and in Table 1 and Table 3 in Additional files. Total genomic DNA was extracted from whole specimens, following the salting out protocol [57]. Sequences were obtained from two mitochondrial gene fragments, the Cytochrome Oxidase subunit I (COI) and 16S rRNA (16S); and from one nuclear fragment, a portion of the rudimentary gene (CAD). For PCR amplifications, the following primer pairs were used: $\mathrm{COI}=\mathrm{LC01490} 5^{\prime}$ - .

TCAACAAATCATAAAGATATTGG-3' HC02198 5'-TAAACTTCAGGGTGACCAA AAAATCA-3' [58]; $16 \mathrm{~S}=16 \mathrm{SA} 5^{\prime}$-CGCCTGTTTATCAAAAACAT- $3{ }^{\prime} ;$; 16SB 5' - CTCCGGTTTGAACTCAGATCA- 3' [59]; CAD = CD439F 5'-TTCAGTGTACARTTYCAYCCHGARCA YAC-3' CD688R 5' '-TGTATACCTAGAGGATCDAC RTTYTCCATRTTRCA-3' [60].

Amplifications of the mitochondrial genes were performed with the following general cycle conditions: initial denaturation at $96^{\circ} \mathrm{C}$ for three minutes, followed by 35 cycles of denaturation at $94{ }^{\circ} \mathrm{C}$ for one minute, annealing at $54^{\circ}-57^{\circ} \mathrm{C}$ for $40 \mathrm{~s}, 1-\mathrm{min}$. Extension at $72^{\circ} \mathrm{C}$ and a last 7-min. Elongation step at $72^{\circ} \mathrm{C}$. Reactions were performed in a $25 \mu \mathrm{l}$ volume containing $\left(\mathrm{NH}_{4}\right)_{2} \mathrm{SO}_{4}$ $16 \mathrm{mM}$, Tris- $\mathrm{HCl} 67 \mathrm{mM}$ (pH 8.8 at $25^{\circ} \mathrm{C}$ ), $\mathrm{MgCl}_{2} 3$ $\mathrm{mM}, 1 \mathrm{mM}$ of each dNTP, $0.8 \mathrm{pmol}$ of each primer and 1.25 units of Taq DNA polymerase. A touchdown PCR protocol was used to amplify the CAD marker with the following thermal cycling conditions: $94{ }^{\circ} \mathrm{C}$ for $3.5 \mathrm{~min}$, followed by 20 cycles of $94{ }^{\circ} \mathrm{C}, 30 \mathrm{~s}$, annealing temperatures stepdowns every cycle of $0.4{ }^{\circ} \mathrm{C}$ (from 58 to $50^{\circ} \mathrm{C}$ ), $35 \mathrm{~s}, 72^{\circ} \mathrm{C}, 2.5 \mathrm{~min}$ and additional 20 cycles of $94^{\circ} \mathrm{C}, 30$ s, $55^{\circ} \mathrm{C}, 35 \mathrm{~s}, 72^{\circ} \mathrm{C}, 2.5 \mathrm{~min}$. We used an MJ MINI Personal Thermal Cycler (BIO-RAD Laboratories, US) and LifeECO Thermal Cycler to perform PCR amplifications. The PCR products were purified with a GENEAID- Gel/ PCR DNA Fragments Extraction Kit and sent to an external sequencing service (Macrogen Inc.: www. macrogen.com). Sequences were edited and aligned with GENEIUS v9.1.6 [61]. A total of 84 new sequences have been deposited in GenBank (Accession numbers COI: MT949495-MT949523; 16S: MT957149-MT957177; CAD: MT966846-MT966871).

\section{Molecular phylogeny and divergence time estimation}

Phylogenetic analyses (BI) were first performed using single-gene alignments and then, Bayesian inference (BI) and Maximum likelihood analysis (ML) were both performed on the concatenated (mtDNA + nucDNA) dataset using, respectively, MRBAYES v3.2.1 [62] and IQTREE [55] as implemented in W-IQ-TREE [63]. The best-fit models for the study data sets proved to be a Generalized Time- Reversible model with a proportion of invariable sites and heterogeneous substitution rates following a gamma distribution $(G T R+I+G)$ for the COI and $16 \mathrm{~S}$ and Generalised Time-Reversible model with a proportion of invariant sites $(G T R+I)$ for CAD. The best fitting model to analyse each partition was selected by JMODELTEST [64] using the Akaike information criterion. The BI analysis was performed by running 5,000,000 generations, with Markov chains sampled every 1000 generations. A 10\% burn-in was applied and the remaining trees were used to compute a $50 \%$ majority rule consensus tree and posterior probabilities. We assessed convergence of the runs by investigating the average standard deviation of split frequencies, potential scale reduction factor (PSRF) in MRBAYES and effective sample size (ESS) of all parameters in TRACER 1.6 [65]. Average standard deviation below 0.01, value of PSRF close to 1.00 and ESS values over 200 for all parameters were acknowledged as good indicators of convergence. A ML phylogenetic reconstruction was performed running 1000 ultrafast bootstrap replications [66] followed by 1000 replications of assessment of branch supports with single branch tests with SH-like approximate likelihood ratio test. The best fitting model to analyze each partition was selected as for BI.

To estimate the relative age of lineage divergences, an uncorrelated lognormal Bayesian molecular relaxed clock model and a Yule process prior were used on the mtDNA data set using the software BEAST v.1.8.2 [67]. Although some fossils ascribed to Meligethinae have been described and dated from Baltic ambers [44, 45], these oldest representatives require a careful revision by other specialists of this group, which consider them either doubtfully attributable to Meligethes or even to the same Meligethinae subfamily. Hence, given the lack of reliable fossil records or useful dated palaeogeographic events to calibrate our trees, we used an average value of the COI substitution rate in a range between 1.5 and $3.54 \%$, which represent the most accurate estimated rate for mitochondrial DNA in coleopterans so far [68-70] and hence widely applied in molecular dating of beetle phylogeography and phylogeny (e.g. 6, 23, [71-75]). Therefore, for the molecular clock analysis we applied an average rate of 0.0126 . The GTR model was transferred to the HKY $[76,77]$ model because there are low ESS values for some parameters in the analyses when 
applying the GTR model. The analysis was independently performed three times, with 100 million generations and sampling of trees every 10,000 steps. Effective Sample Size (ESS) was evaluated in Tracer v1.6 [65], considering runs with ESS values above 200. Output trees were generated in Tree Annotator v1.8.2 (BEAST package), using maximum clade credibility (MCC) after a $10 \%$ burn-in and median heights.

\section{Supplementary Information}

The online version contains supplementary material available at https://doi. org/10.1186/s12983-021-00390-4.

Additional file 1. Abbreviations. (Museum acronyms following Evenhuis NL. The Insect and Spider Collections of the World Website. 2020http:// hbs.bishopmuseum.org/codens/ [accessed at March 26th, 2020]).

Additional file 2 Table 3 List of morphological and bionomical characters used in the cladistic analysis on all 63 Meligethes s.l. [Meligethes s.str. + Odonthogethes] species and on selected outgroup species, including Brassicogethes.

Additional file $\mathbf{3}$ Table 4 Matrix of the character states in the 63 Meligethes s.l. species and in the selected purported 7 outgroup species and genera, including Brassicogethes.

Additional file $\mathbf{4}$ The Bayesian tree of Meligethes-complex on analyses of the mitochondrial COl gene. The posterior probabilities exceeding 50\% are shown at nodes.

Additional file $\mathbf{5}$ The Bayesian tree of Meligethes-complex on analyses of the mitochondrial 165 gene. The posterior probabilities exceeding $50 \%$ are shown at nodes

Additional file $\mathbf{6}$ The Bayesian tree of Meligethes-complex on analyses of the nuclear CAD gene. The posterior probabilities exceeding 50\% are shown at nodes.

Additional file $\mathbf{7}$ The Bayesian tree of Meligethes-complex based on combined molecular and morphological dataset. The posterior probabilities exceeding 50\% are shown at nodes.

Additional file 8 The Maximum Likelihood (ML) tree of Meligethescomplex based on morphological data, performed by IQ-TREE using default settings.

\section{Acknowledgements}

We would like to thank the curators of the following institutions and some private entomologists for loaning valuable specimens necessary to complete our phylogenetic study: J. Jelínek (NMPC), A. Lasoń (CAL), M. Barklay (NHMUK), T. Deuve (MNHN), M. Danielsson (MZLU), F. Hieke (ZMHB), O. Merkl (HNHM), M. Jäch and H. Schilhammer (NHMW), E. Sprecher (NHMB), I. Löbl (MHNG), A.G. Kirejtshuk (ZIN), and N. B. Nikitsky (ZMUM). Special thanks are due to our colleague Sadatomo Hisamatsu (Matsuyama, Japan), for his valuable help in providing us with precious material of Meligethes spp. from Japan and Taiwan. We greatly appreciated the valuable comments on an earlier version of this manuscript received from our dear friend and colleague Josef Jelínek, Department of Entomology, National Museum, Horní Počernice, Czech Republic, and from two anonymous revievers. The authors greatly thank also the colleague Kirill Makarov (Moscow, Russia) for authorization to use his picture of Meligethes flavimanus (Fig. 1 a (a)), as well as our friend Lec Borowiec (Wrocław, Poland) for authorization to use his picture of Odonthogethes denticulatus (Fig. 1 a (b)). Special thanks are also due to N. Falchi (Rome), for preparation of the Fig. 1 a (c)). The authors are grateful to Prof. Xinfen Gao, Curator of the Herbarium (CDBI), Chengdu Institute of Biology, Chinese Academy of Sciences, and to the Chinese amateur botanist Shuanlu Dong (Meixian, Shaanxi) for cooperation and assistance in identifying several host plants.

\section{Authors' contributions}

PA, SS, ML and $\mathrm{MH}$ designed the project, SS, PA, DB, ML, SP and AS analyzed the data and prepared graphs and illustrations, PA, ARC, SS, MT, ML, DB and
EM wrote the manuscript, ML, MH and PA contributed materials and resources. All authors contributed to the final manuscript.

\section{Funding}

This paper was partly supported by funds from the National Natural Science Foundation of China (No. 32000321) ML and funds to PA and SS from the Italian Ministero dell'Università e della Ricerca. This paper was also partly supported by funds from the Fundamental Research Funds for Chinese Central Universities (Z109021305) MH, and the senior author (ML) thanks the China Scholarship Council for financial support for her stay in Rome (November, 2016-December, 2017), in collaboration with coauthor P.A.

\section{Availability of data and materials}

Sequence data (mtDNA and nDNA) can be found in GenBank (see Table 2). Material of Meligethinae studied for the aims of the present research was examined from or is preserved in the institutions and private collections listed above under Abbreviations.

Ethics approval and consent to participate

Not applicable.

\section{Consent for publication}

Not applicable.

\section{Competing interests}

The authors declare to have not competing interests.

\section{Author details}

${ }^{1}$ Key Laboratory of Plant Protection Resources and Pest Management of Ministry of Education, Entomological Museum, Northwest A\&F University, Yangling, Xianyang, Shaanxi, China. ${ }^{2}$ College of Agriculture, Yangtze University, Jingzhou 434025, Hubei, China. ${ }^{3}$ California Department of Food \& Agriculture, Plant Pest Diagnostics Center, Sacramento, CA, USA.

4Dipartimento di Biologia e Biotecnologie "Charles Darwin", Sapienza Università di Roma, Rome, Italy.

Received: 24 June 2020 Accepted: 12 February 2021

Published online: 06 March 2021

\section{References}

1. Coleoptera AP, Kateretidae N. Fauna d'Italia. Bologna: Calderini Edizioni; 1993

2. Audisio P, Cline AR, De Biase A, Antonini G, Mancini E, Trizzino M, Costantin L, Strika S, Lamanna F, Cerretti P. Preliminary re-examination of genus-level taxonomy of the pollen beetle subfamily Meligethinae (Coleoptera: Nitidulidae). Acta Ent Mus N Pragae. 2009;49:341-504.

3. Jelínek J, Carlton CE, Cline AR, Leschen RAB. 10.26. Nitidulidae Latreille. Berlin: Walter De Gruyter; 2010.

4. Cline AR, Smith TR, Miller K, Moulton M, Whiting M, Audisio P. Molecular phylogeny of Nitidulidae: assessment of subfamilial and tribal classification and formalization of the family Cybocephalidae (Coleoptera: Cucujoidea). Syst Entomol. 2014:39:758-72.

5. Liu M, Sabatelli S, Mancini E, Trizzino M, Huang M, Cline AR, Audisio P. Rediscovery of Brassicogethes salvan (Coleoptera: Nitidulidae, Meligethinae) in the southwestern Alps. Ins Cons Div. 2020;12:80-7.

6. Liu M, Huang M, Cline AR, Cardoli P, Audisio P, Sabatelli S. Re-examination of the genus-level taxonomy of the pollen beetle subfamily Meligethinae part 1. Sagittogethes Audisio \& Cline 2009 and allied genera; with description of a new genus (Coleoptera: Nitidulidae). Fragm entomol. 2020; 52(1):119-35.

7. Audisio P, Sabatelli S, Jelínek J. Revision of the pollen beetle genus Meligethes (Coleoptera: Nitidulidae). Fragm entomol. 2015;46:19-112.

8. Liu M, Yang $X$, Huang $M$, Jelínek J, Audisio P. Four new species of Meligethes Stephens from China and additional data on other species of the genus (Coleoptera: Nitidulidae: Meligethinae). Zootaxa. 2016;4121: 101-16.

9. Liu M, Huang M, Cline AR, Sabatelli S, Audisio P. A new species of Meligethes Stephens from China and additional data on members of the M. chinensis species-complex (Coleoptera: Nitidulidae, Meligethinae). Fragm entomol. 2017:49:79-84 
10. Liu M, Huang M, Cline AR, Audisio P. New and poorly known Meligethes Stephens from China, with bionomical data on some species (Coleoptera: Nitidulidae: Meligethinae). Zootaxa. 2018;4392:546-66.

11. Ormerod EA. Life history of Meligethes. Ent mon Mag. 1874;11:46-52.

12. Hayashi N. A contribution to the knowledge of the larvae of Nitidulidae occurring in Japan (Coleoptera: Cucujoidea). Ins Matsum. 1978;14:1-97.

13. Hisamatsu S-T. Revision of the Meligethinae of Taiwan (Coleoptera, Nitidulidae). Japan J Syst Ent. 2009;15:17-46.

14. Audisio P, De Biase A, Romanelli P, Angelici MC, Ketmaier V, De Matthaeis E. Molecular re-examination of the taxonomy of the Meligethes viridescens species complex (Coleoptera: Nitidulidae). Biochem Syst Ecol. 1999;28:1-13.

15. Audisio P, Belfiore C, De Biase A, Antonini G. Identification of Meligethes matronalis Audisio and Spornraft, 1990 and M. subaeneus Sturm, 1845, based on morphometric and bionomic characters (Coleoptera: Nitidulidae). Eur J Ent. 2001;98:87-97.

16. Audisio P, De Biase A, Antonini G, Belfiore C, Oliverio M. Morphological, molecular, and ecological evidence of a new euro-Anatolian species of the Meligethes coracinus complex (Coleoptera: Nitidulidae). Ins Syst Evol. 2001; 31:361-85.

17. Audisio P, De Biase A, Antonini G, Oliverio M, Ketmaier V, De Matthaeis E. Specific distinction by allozymic data of sympatric sibling species of the pollen beetle genus Meligethes (Coleoptera: Nitidulidae). It J Zool. 2002;69: 65-9.

18. Audisio P, De Biase A, Antonini G. A new exceptional Meligethes of the M. aeneus species-group from Western Alps and an updated key to identification of M. aeneus and allied species (Coleoptera: Nitidulidae: Meligethinae). Ins Syst Evol. 2003;34:121-30.

19. Audisio P, De Biase A, Antonini G, Mancini E, Özbek H, Gultekin L. Redescription and natural history of Meligethes longulus Schilsky, 1894, and provisional revision of the M. coracinus species-complex (Coleoptera, Nitidulidae, Meligethinae). It J Zool. 2005;72:73-85.

20. Audisio P, Mancini E, De Biase A. A new species of the pollen-beetle genus Meligethes (Coleoptera: Nitidulidae) of the M. aeneus group from Greece, with review of the M. subaeneus complex. Zootaxa. 2006;1275:43-60.

21. Audisio P, Cline AR, Mancini E, Trizzino M, Avgin SS, De Biase A. Four new Palaearctic Brassicogethes (Coleoptera, Nitidulidae, Meligethinae), and phylogenetic inference on the B. coracinus group. Rend Lincei. 2011;22:235-68.

22. De Biase A, Antonini G, Mancini E, Trizzino M, Cline A, Audisio P. Discordant patterns in the genetic, ecological, and morphological diversification of a recently radiated phytophagous beetle clade (Coleoptera: Nitidulidae: Meligethinae). Rend Lincei. 2012;23:207-15.

23. Mancini E, De Biase AD, Cline AR, Antonini G, Trizzino M, Clayhills T, Sabatelli S, Cerretti P, Audisio P. Morphological, genetic and host-plant diversification in pollen-beetles of the Brassicogethes coracinus group (Coleoptera: Nitidulidae: Meligethinae). Rend Lincei. 2016;27:321-39.

24. Trizzino M, Audisio P, Antonini G, De Biase A, Mancini E. Comparative analysis of sequences and secondary structures of the rRNA internal transcribed spacer 2 (ITS2) in pollen beetles of the subfamily Meligethinae (Coleoptera, Nitidulidae): potential use of slippage-derived sequences in molecular systematics. Mol Phylogenet Evol. 2009;51:215-26.

25. Audisio P, Jelínek J, Cline AR, Mancini E, Trizzino M, Cerretti P, Antonini G. Description and taxonomic position of a new genus and species of southern African pollen beetle (Coleoptera: Nitidulidae: Meligethinae). Zootaxa. 2011;2927:49-56.

26. Audisio P, Cline AR, Solano E, Mancini E, Lamanna F, Antonini G, Trizzino M. A peculiar new genus and species of pollen-beetle (Coleoptera, Nitidulidae) from eastern Africa, with a molecular phylogeny of related Meligethinae. Syst Biodivers. 2014;12:77-91.

27. Stephens JF. Illustrations of British entomology; or, a synopsis of indigenous insects: containing their generic and specific distinctions; with an account of their metamorphoses, times of appearance, localities, food, and economy, as far as practicable. Mandibulata: Baldwin \& Cradock, London; 1830.

28. Olivier AG. Entomologie, ou histoire naturelle des insectes, aves leurs caractères génériques et spécifiques, leur description, leur synonymie, et leur figure enluminée. Coléoptères. Tome second: de Baudouin, Paris; 1790.

29. Marsham T. Entomologia Britannica, sistens insecta britanniae indigena, secundum methodum linnaeanam disposita. Tomus I. Coleoptera. Londini: Wilks et Taylor; 1802

30. Heer O. Fauna Coleopterorum Helvetica. Pars 1 (3). Turici: Orelii, Fuesslini et Sociorum; 1841. p. 361-652.
31. Rebmann O. Revision der Gattung Meligethes (Col. Nitid.). Die paläarktischen Arten der Gruppe des M. atratus OI. (7. Beitrag zur Kenntnis der Nitiduliden.). Ent BI Zeitschr Biol und Syst Käf. 1956;52:124-35.

32. Rebmann O. Revision der Gattung Meligethes Subgenus Odonthogethes (Col. Nitid.) (6. Beitrag zur Kenntnis der Nitiduliden). Ent BI Zeitschr Biol und Syst Käf. 1956;52:42-8.

33. Chen $Y$, Kirejtshuk AG, Huang M. A new species of the subgenus Meligethes Stephens (Coleptera: Nitidulidae) from Sichuan. China Entomotaxonomia. 2013;35:35-40.

34. Chen $Y$, Lin $X$, Huang $M$, Yang XK. A new species of Lamiogethes and a new species of Meligethes from China (Coleoptera: Nitidulidae: Meligethinae). Zootaxa. 2015;3999:413-20.

35. Potter D, Eriksson T, Evans RC, Oh S, Smedmark JEE, Morgan DR, Kerr M, Robertson KR, Arsenault M, Dickinson TA. Phylogeny and classification of Rosaceae. Plant Syst Evol. 2007;266:5-43.

36. Potter D, Still SM, Grebenc T, Ballian D, Božič G, Franjiæ J, Kraigher H. Phylogenetic relationships in tribe Spiraeeae (Rosaceae) inferred from nucleotide sequence data. Plant Syst Evol. 2007;266:105-18.

37. Audisio P, Cline AR, Lamanna F, Trizzino M, Antonini G, Mancini E, De Biase A. Revision of the southern African pollen beetle genus Anthystrix (Coleoptera: Nitidulidae: Meligethinae). Ann Entomol Soc Am. 2009;102:9981012.

38. Audisio P, Cline AR, Trizzino M, Mancini E, Antonini G, Sabatelli S, Cerretti P. Revision of the African pollen beetle genera Tarchonanthogethes and Xenostrongylogethes, with insect-host plant relationships, identification key, and cladistic analysis of the Anthystrix genus-complex (Coleoptera: Nitidulidae: Meligethinae). Zootaxa. 2015;3920:101-52.

39. Alice LA, Campbell CS. Phylogeny of Rubus (Rosaceae) based on nuclear ribosomal DNA internal transcribed spacer region sequences. Am J Bot. 1999:86:81-97.

40. Fougère-Danezan M, Joly S, Bruneau A, Gao XF, Zhang LB. Phylogeny and biogeography of wild roses with specific attention to polyploids. Ann Bot London. 2014;115:275-91.

41. Wang Y, Chen Q, Chen T, Tang H, Liu L, Wang X. Phylogenetic insights into Chinese Rubus (Rosaceae) from multiple chloroplast and nuclear DNAs. Front PI Sci. 2016:7:968.

42. Lu LD, Gu CZ, Li CL, Crinan A, Bartholomew B, Brach AR, Boufford DE, Ikeda $\mathrm{H}$, Ohba H, Robertson KR, Spongberg SA. Rosaceae. In: Wu ZY, Ravan PH, Hong DY, editors. Flora of China, vol. 9. Beijing/St. Louis: Science Press/ Missouri Botanical Garden Press; 2003. p. 46-434.

43. Sabatelli S, Liu M, Cline AR, Lasoń A, Macuvele S, Muambalo K, Chuquela L, Audisio P. Palms and pollen beetles: two new anthophilous beetle species of Meligethinus from Mozambique (Coleoptera: Nitiduldae: Meligethinae). Zootaxa. 2020;4802(1):032-40.

44. Kirejtshuk AG, Poinar J Jr. Species of two Paleoendemic sap beetle genera of the tribe Nitidulini (Nitidulidae: Coleoptera) from the Baltic and Dominican Amber. Paleontol J. 2007:41(6):629-41.

45. Kirejtshuk AG. The oldest representatives of the subfamilies Meligethinae (Coleoptera: Nitidulidae) and Brontinae (Coleoptera: Silvanidae) from Baltic amber and some evolutionary notes. Pol J Ent. 2011;80:729-45.

46. Hipsley CA, Müller J. Beyond fossil calibrations: realities of molecular clock practices in evolutionary biology. Front Genet. 2014;26:5-138.

47. Cutter AD. Divergence times in Caenorhabditis and Drosophila inferred from direct estimates of the neutral mutation rate. Mol Biol Evol. 2008;25(4): 778-86.

48. Xiang Y, Huang CH, Hu Y, Wen J, Li S, Yi T, Chen H, Xiang J, Ma H. Evolution of Rosaceae fruit types based on nuclear phylogeny in the context of geological times and genome duplication. Mol Biol Evol. 2017;34:262-81.

49. Ehrlich PR, Raven PH. Butterflies and plants: a study in coevolution. Evolution. 1964;18:586-608.

50. Jermy T. Insect-Host-plant Relationship - Co-evolution or Sequential Evolution? In: Jermy T, editor. The Host-Plant in Relation to Insect Behaviour and Reproduction; 1976

51. Fang JY, Song J-C, Liu H-J, Piao S-L. Vegetation-climate relationship and its application in the division of vegetation zones in China. Acta Bot Sin. 2002; 44:1105-22.

52. Sabatelli S, Audisio P, Jelínek J, Liu M, Huang M. Description of the unknown males of three Chinese Meligethes (Coleoptera: Nitidulidae, Meligethinae). Fragm entomol. 2020;52(2):351-3.

53. Goloboff PA, Catalano SA. TNT version 1.5, including a full implementation of phylogenetic morphometrics [J]. Cladistics. 2016;32:221-38. 
54. Nixon KC. Winclada, Version 1.00.08. Published by the Author, Ithaca, New York. [WWW document]. 2002;http://www.cladistics.com. [accessed on January 2017, no longer available].

55. Nguyen L-T, Schmidt HA, Haeseler A, Minh BQ. IQ-TREE: a fast and effective stochastic algorithm for estimating maximum-likelihood phylogenies. Mol Biol Evol. 2015;32:268-74.

56. C Maddison WP, Maddison DR. Mesquite: A modular system for evolutionary analysis, version 2.74. 2010;http://mesquiteproject.org.

57. Aljanabi SM, Martinez I. Universal and rapid salt-extraction of high quality genomic DNA for PCR-based techniques. Nucl Ac Res. 1997;25:4692-3.

58. Folmer O, Black M, Hoeh W, Lutz R, Vrijenhoek R. DNA primers for amplification of mitochondrial cytochrome $\mathrm{C}$ oxidase subunit I from diverse metazoan invertebrates. Mol Mar Biol Biotechnol. 1994;3(5):294-9.

59. Simon C, Frati F, Beckenbach A, Crespi B, Liu H, Flook P. Evolution, weighting, and phylogenetic utility of mitochondrial gene sequences and a compilation of conserved polymerase chain reaction primers. Ann Entomol Soc Am. 1994;87:651-701.

60. Wild AL, Maddison DR. Evaluating nuclear protein-coding genes for phylogenetic utility in beetles. Mol Phylogenet Evol. 2008:48:877-91.

61. Kearse M, Moir R, Wilson A, Stones-Havas S, Cheung M, Sturrock S, Buxton S, Cooper A, Markowitz S, Duran C, Thierer T, Ashton B, Meintjes P, Drummond A. Geneious basic: an integrated and extendable desktop software platform for the organization and analysis of sequence data. Bioinformatics. 2012;28:1647-9.

62. Huelsenbeck JP, Ronquist F. MRBAYES: Bayesian inference of phylogenetic trees. Bioinformatics. 2001;17:754-5.

63. Trifinopoulos J, Nguyen L-T, von Haeseler A, Minh BQ. W-IQ-TREE: a fast online phylogenetic tool for maximum likelihood analysis. Nucl Ac Res. 2016;44(W1):W232-5.

64. Posada D. jModelTest: phylogenetic model averaging. Mol Phylogenet Evol. 2008;25:1253-6.

65. Rambaut A, Suchard M, Xie D, Drummond A. Tracer v1. 6. 2014; Retrieved from http://tree.bio.ed.ac.uk/software/tracer.

66. Minh BQ, Nguyen MAT, von Haeseler A. Ultrafast approximation for phylogenetic bootstrap. Mol Biol Evol. 2013;30:1188-95.

67. Drummond AJ, Suchard MA, Xie D, Rambaut A. Bayesian phylogenetics with BEAUti and the BEAST 1.7. Mol Biol Evol. 2012;29:1969-73.

68. Farrell BD. Evolutionary assembly of the milkweed fauna: cytochrome oxidase I and the age of Tetraopes beetles. Mol Phylogenet Evol. 2001;18:467-78.

69. Papadopoulou A, Anastasiou I, Vogler AP. Revisiting the insect mitochondrial molecular clock: the mid-Aegean trench calibration. Mol Phylogenet Evol. 2010;27:1659-72.

70. Pons J, Ribera I, Bertranpetit J, Balke M. Nucleotide substitution rates for the full set of mitochondrial protein-coding genes in Coleoptera. Mol Phylogenet Evol. 2010;56:796-807.

71. Sabatelli S, Audisio P, Antonini G, Solano E, Martinoli A, Trizzino M. Molecular ecology and phylogenetics of the water beetle genus Ochthebius revealed multiple independent shifts to marine rockpools lifestyle. Zool Scr. 2016;45(2):175-86.

72. Grebennikov W. Phylogeography and sister group of Lupangus, a new genus for three new flightless allopatric forest litter weevils endemic to the eastern Arc Mountains, Tanzania (Coleoptera: Curculionidae, Molytinae). Fragm entomol. 2017:49:37-55.

73. Kawakami Y, Yamazaki K, Ohashi K, Nakahama N. Genetic structure, diversity and demography based on mitochondrial DNA analysis of Cheilomenes sexmaculata (Coleoptera: Coccinellidae). Entomol Sci. 2020;23:385-92.

74. Riccieri A, Mancini E, Salvi D, Bologna MA. Phylogeny, biogeography and systematics of the hyper-diverse blister beetle genus Hycleus (Coleoptera: Meloidae). Mol Phylogenet Evol. 2020;144:106706.

75. Sabatelli S, Liu M, Badano D, Mancini E, Trizzino M, Cline AR, Endrestøl A, Huang M, Audisio P. Molecular phylogeny and host-plant use (Lamiaceae) of the Thymogethes pollen beetles (Coleoptera). Zool Scr. 2020;49:28-46.

76. Rodríguez F, Oliver JL, Marín A, Medina JR. The general stochastic model of nucleotide substitution. J Theor Biol. 1990;142:485-501.

77. Hasegawa M, lida Y, Yano TA, Takaiwa F, Iwabuchi M. Phylogenetic relationships among eukaryotic kingdoms inferred from ribosomal RNA sequences. J Mol Evol. 1985;22(1):32-8.

\section{Publisher's Note}

Springer Nature remains neutral with regard to jurisdictional claims in published maps and institutional affiliations.

\section{Ready to submit your research? Choose BMC and benefit from}

- fast, convenient online submission

- thorough peer review by experienced researchers in your field

- rapid publication on acceptance

- support for research data, including large and complex data types

- gold Open Access which fosters wider collaboration and increased citations

- maximum visibility for your research: over $100 \mathrm{M}$ website views per year

At $\mathrm{BMC}$, research is always in progress.

Learn more biomedcentral.com/submissions 